\title{
Неклассическая логика
}

Non-classical Logic

\section{Л.Ю. ДЕвяткин \\ О выразительных возможностях отдельных расширений четырехзначной логики Белнапа}

\author{
Леонид Юрьевич Девяткин \\ Институт философии РАН. \\ Российская Федерация, 109240, г. Москва, ул. Гончарная, д. 12, стр. 1. \\ E-mail: deviatkin@iph.ras.ru
}

Аннотация: Статья посвящена замкнутым классам функций четырехзначной логики. Мы представляем следующие результаты.

(1) Базовые операции логики, полученной расширением четырехзначной алгебры Де Моргана оператором конфляции, порождают замкнутый класс всех функций, которые одновременно сохраняют классические истинностные значения и самодвойственны относительно конфляции. Этот класс предполон в классе всех функций, сохраняющих классические истинностные значения.

(2) Между замкнутым классом, порожденным базовыми операциями логики истины фон Вригта и классом всех функций, сохраняющих классические истинностные значения, лежит в точности два замкнутых класса. Каждый из них представляет собой класс всех функций, одновременно сохраняющих классические истинностные значения и одно из трехэлементных надмножеств множества классических истинностных значений.

(3) Базовые операции тетравалентной модальной логики, полученной расширением четырехзначной алгебры Де Моргана оператором необходимости, порождают замкнутый класс всех функций, которые одновременно сохраняют классические истинностные значения, самодвойственны относительно конфляции, а также сохраняют оба трехэлементных надмножества множества классических истинностных значений. Мы показываем, что данный класс предполон в классе всех функций, которые одновременно сохраняют классические истинностные значения и самодвойственны относительно конфляции. Кроме того, мы демонстрируем, что между этим классом и замкнутым классом, порожденным операциями логики истины фон Вригта, находится в точности один замкнутый класс.

Таким образом, мы получаем семиэлементную решетку, состоящую из всех возможных четырехзначных расширений тетравалентной модальной логики, которые сохраняют классические истинностные значения.

Ключевые слова: алгебра Де Моргана, четырехзначная логика Белнапа, логика истины фон Вригта, логика истины Tr, тетравалентная модальная логика, замкнутые классы функций

(c) Девяткин Л.Ю. 
Для цитирования: Девяткин Л.Ю. О выразительных возможностях отдельных расширений четырехзначной логики Белнапа // Логические исследования / Logical Investigations. 2020. T. 26. № 2. C. 116-143. DOI: 10.21146/2074-1472-2020-26-2-116-143

\section{Введение}

Многозначные логики выступают полезным инструментом во многих областях современных логических исследований. В числе наиболее известных многозначных логик находится «полезная четырехзначная логика» Н. Белнапа [Belnap, 1977], которую мы будем обозначать В $\mathbf{B}_{4}$. Как отмечает А.С. Карпенко |Карпенко, 2015, §5], В 4 оказалась удобной «базовой» системой, обогащая которую можно получить интересные новые логики. Существует обширная литература, посвященная логикам, которые являются расширениями FDE. Особую роль играют расширения $\mathbf{B}_{4}$, полученные с помощью пополнения языка этой логики новыми истинностнофункциональными операторами, такими как, например, модальные операторы, оператор формальной противоречивости, импликация и отрицание, обладающие свойствами, характерными для данных связок в классической логике. Обстоятельный обзор подобных расширений доступен в Petrukhin, Shangin, 2019].

А.С. Карпенко принадлежит серия работ, посвященных выразительным возможностям отдельных четырехзначных языковых расширений $\mathbf{B}_{4}$ Karpenko, 2013; Карпенко, 2015; Karpenko, 2017. Эта работа лежит в русле указанных исследований. Основной задачей является проверка выдвинутой в [Karpenko, 2017] гипотезы о выразительных возможностях языков «логики истины» $\mathbf{T}^{\prime \prime}$ Г.Х. фон Вригта и логики $\mathbf{T r}$, полученной добавлением к связкам $\mathbf{B}_{4}$ оператора «конфляции» ${ }^{1}$. Методы исследования близки к таковым в работах А. Аврона и О. Ариэли, посвященных выразитель-

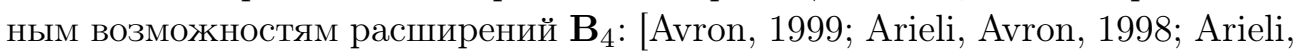
Avron, 2017]. Мы анализируем замкнутые классы функций на множестве $\{\mathbf{t}, \mathbf{b}, \mathbf{n}, \mathbf{f}\}$, порожденные наборами базовых операций интересующих нас расширений $\mathbf{B}_{4}$. Это требует уточнения.

Обычно многозначные логики определяются с помощью логических матриц. Логическая матрица представляет собой структуру, состоящую из алгебры и подмножества $D$ множества-носителя этой алгебры, называемого классом выделенных значений. Матрицы с совпадающими алгебрами и различающимися классами выделенных значений порождают различающиеся логики. В матрице четырехзначной логики Белнапа используется алгебра Де Моргана и два выделенных значения

\footnotetext{
${ }^{1}$ Необходимые формальные определения будут даны ниже.
} 
(см. Omori, Wansing, 2017, §2.1]). Но логические матрицы, базирующиеся на алгебре Де Моргана, могут иметь и другое число выделенных значений: одно или три. Основанные на алгебре Де Моргана логики с одним выделенным значением описываются в [Karpenko, 2017]. Вопрос о свойствах четырехзначной логики Белнапа с расширенным или суженным классом выделенных значений специально исследуется в [Shramko et al., 2017; Shramko et al., 2019.

В настоящей работе мы рассматриваем алгебры вне связи с классами выделенных значений. Таким образом, полученные результаты носят обобщенный характер. Они касаются не только четырехзначных расширений $\mathbf{B}_{4}$ с двумя выделенными значениями, но и родственных систем с однм и тремя выделенными значениями.

Дальнейшая структура работы такова. В оставшейся части Введения мы даем базовые определения, необходимые для точной постановки основной задачи исследования, и осуществляем такую постановку. В Разделе 1 исследуются свойства замкнутого класса функций $\mathrm{Tr}$, порожденного базовыми операциями логики $\operatorname{Tr}$. В Разделе 2 исследуются свойства замкнутого класса функций $T^{\prime \prime}$, порожденного базовыми операциями логики $\mathbf{T}^{\prime \prime}$. В Разделе 3 мы показываем, что замкнутый класс $T M L$, порожденный операциями четырехзначной модальной логики TML [Карпенко, 2015, §7], представляет собой наибольший общий замкнутый подкласс $\operatorname{Tr}$ и $T^{\prime \prime}$, и исследуем свойства $T M L$. В Заключении мы даем общее резюме полученных результатов, главным из которых является полное описание решетки замкнутых классов функций, лежащих между $T M L$ и $\ell_{4}-$ замкнутым классом функций, порожденным операциями четырехзначной логики Лукасевича.

Перейдем к формальной постановке целей и задач исследования. Приведенные ниже определения заимствуем из книги Марченков, 2004, С. 812]. Там же можно найти более подробное изложение данного материала.

Определение 1 (Реализация функции формулой). Пусть $R-$ непустое множество функций, заданных на некотором множестве. Каждой функции из этого множества сопоставим синтаксическую конструкцию, которую будем называть формулой над $R$. Пусть символом $f$ обозначена функция из $R$ от $n$ переменных, а $x_{1}, \ldots, x_{n}-$ символы переменных. Тогда выражение $f\left(x_{1}, \ldots, x_{n}\right)$ - формула над $R$. Формуле $f\left(x_{1}, \ldots, x_{n}\right)$ сопоставляем ту функцию из $R$, которая имеет обозначение $f$, и говорим, что функция $f$ реализуется формулой $f\left(x_{1}, \ldots, x_{n}\right)$. Так как любой функции из $R$ можно сопоставить формулу над $R$ описанным выше способом, также верно, что любая функция из $R$ реализуется некоторой формулой над $R$. 
Пусть символом $g$ обозначена функция из $R$ от $m$ переменных, а $\Phi_{1}, \ldots, \Phi_{m}$ - формулы над $R$ или символы переменных (необязательно различные). В этом случае $g\left(\Phi_{1}, \ldots, \Phi_{m}\right)-\oint$ фомула над $R$. Допустим, что выражениям $\Phi_{i}$, не являющимся символами переменных, сопоставлены функции $f_{i}$. Выражениям $\Phi_{j}$, которые суть символы переменных $x_{p(j)}$, сопоставим функции $f_{j}\left(x_{p(j)}\right)$, где $f_{j}\left(x_{p(j)}\right)=x_{p(j)}$. В этом случае формуле $g\left(\Phi_{1}, \ldots, \Phi_{m}\right)$ сопоставляем функцию $g\left(f_{1}, \ldots, f_{m}\right)$, реализуемую этой формулой.

Определение 2 (Суперпозиция). Если функция $f$ реализуется формулой, которая содержит только символы функций $f_{1}, \ldots, f_{s}$, а также символы переменных, то говорим, что функция $f$ является суперпозицией функций $f_{1}, \ldots, f_{s}$, или что $f$ получена из функций $f_{1}, \ldots, f_{s}$ с помощью операции суперпозичии.

Определение 3 (Замкнутость). Пусть $R$ - произвольное множество функций, заданных на некотором множестве. Замыканием $R$ называем множество $[R]$ всех функций, которые являются суперпозициями функций из $R$. Если $[R]$ - замыкание $R$, называем $R$ порождающей системой $[R]$. Говорим, что множество функций $R$ (функционально) замкнуто, если $R=[R]$. Множества такого рода также будем называть замкнутыми классами.

Определение 4 (Предполнота). Замкнутый класс $Q$ предполон в замкнутом классе $R$, если для всех таких $g$, что $g \in R$ и $g \notin Q$, имеет место $[Q \cup\{g\}]=R$.

Предметом настоящего исследования являются отношения между замкнутыми классами функций на множестве $\{\mathbf{t}, \mathbf{b}, \mathbf{n}, \mathbf{f}\}$, порожденными следующими системами операций: $\{\vee, \wedge, \neg\},\left\{\wedge, \vee, \neg, e_{2}\right\},\{\wedge, \vee, \neg,-\}$, $\{\wedge, \vee, \neg,-, \supset\}$. Табличные определения соответствующих операций таковы:

\begin{tabular}{|c|c|c|c|c|c|c|c|c|c|c|}
\hline$\wedge$ & $\mathrm{t}$ & b & $\mathbf{n}$ & f & & & & $\mathrm{f}$ & $x$ & $\neg x$ \\
\hline $\mathrm{t}$ & $t$ & b & $\mathrm{n}$ & f & & & & $\mathrm{t}$ & $\mathrm{t}$ & f \\
\hline b & b & b & f & f & & & & b & b & b \\
\hline n & $\mathrm{n}$ & f & $\mathbf{n}$ & f & & & & n & $\mathbf{n}$ & $\mathrm{n}$ \\
\hline $\mathrm{f}$ & f & f & f & f & & & & $\mathrm{f}$ & f & $\mathrm{t}$ \\
\hline & $\supset$ & $\mathrm{t}$ & b & $\mathrm{n}$ & $\mathrm{f}$ & $-x$ & & $x$ & $e_{2}(x)$ & \\
\hline & $\mathrm{t}$ & $\mathbf{t}$ & b & $\mathbf{n}$ & $\mathrm{f}$ & t & & $\mathrm{t}$ & $\mathrm{t}$ & \\
\hline & b & $\mathrm{t}$ & b & $\mathbf{n}$ & $\mathrm{f}$ & $\mathrm{n}$ & & b & $\mathrm{t}$ & \\
\hline & $\mathbf{n}$ & $\mathrm{t}$ & $\mathrm{t}$ & $\mathrm{t}$ & t & b & & $\mathbf{n}$ & f & \\
\hline & f & $\mathbf{t}$ & $\mathbf{t}$ & t & $\mathrm{t}$ & f & & f & $\mathrm{f}$ & \\
\hline
\end{tabular}


Следуя [Karpenko, 2017], примем следующие обозначения: DM4= $[\{\vee, \wedge, \neg\}] ; T^{\prime \prime}=\left[\left\{\wedge, \vee, \neg, e_{2}\right\}\right] ; \operatorname{Tr}=[\{\wedge, \vee, \neg,-\}]$. Кроме того, полагаем $E_{4}=[\{\wedge, \vee, \neg,-, \supset\}]$. В последнем случае мы заменяем порождающую систему для $E_{4}$ из работы А.С. Карпенко на эквивалентную, но более для нас удобную, которую заимствуем из работы Avron, 1999, Th. 3.15]. Интересующие нас наборы операций также рассматриваются в Omori, Sano, 2015 с другими обозначениями: $D M 4-\mathcal{B D}, T^{\prime \prime}-\mathcal{B D}-, \operatorname{Tr}-\mathcal{B D} \Delta$.

Исследованию сравнительных свойств $D M 4, T^{\prime \prime}, \operatorname{Tr}$ и $E_{4}$ специально посвящены работы Karpenko, 2013], [Карпенко, 2015], [Karpenko, 2017]. В них описаны известные в литературе четырехзначные логики, наборы базовых операций которых порождают эти замкнутые классы. Автор указывает на следующие факты: $\operatorname{Tr} \nsubseteq T^{\prime \prime}, T^{\prime \prime} \nsubseteq \operatorname{Tr}, \operatorname{Tr} \subset E_{4}, T^{\prime \prime} \subset E_{4}$. Однако остается открытым вопрос о том, существует ли такой замкнутый класс $K$, что $\operatorname{Tr} \subset K \subset \iota_{4}$ или $T^{\prime \prime} \subset K \subset \iota_{4}$. В этой связи А.С. Карпенко выдвинул следующую гипотезу [Karpenko, 2017].

Классы операций $T^{\prime \prime}$ и $T r$ предполны в $\ell_{4}$.

Иными словами, не существует замкнутых классов, которые были бы собственными надклассами $T^{\prime \prime}$ или $T r$ и одновременно собственными подклассами $E_{4}$. Основная задача нашего исследования состоит в проверке данной гипотезы. Ее решение осуществляется в следующих двух разделах. Первый из них посвящен вопросу о предполноте $\operatorname{Tr}$ в $E_{4}$. Второй - предполноте $T^{\prime \prime}$ в $E_{4}$. Еще одна задача работы состоит в поиске наибольшего общего замкнутого подкласса $\operatorname{Tr}$ и $T^{\prime \prime}$, а также разрешения вопроса о его предполноте в $\operatorname{Tr}$ и $T^{\prime \prime}$. Решению этой задачи посвящен третий раздел.

\section{1. Предполнота $\operatorname{Tr}$ в $t_{4}$}

В этом разделе мы покажем, что $T r$ предполон в $L_{4}$. Нам потребуются дополнительныне определения.

Определение 5 (Сохранение множества функцией). Говорим, что функция $f$, определенная на некотором надмножестве множества $A$, сохраняет множество $A$ (иначе - $A$-замкнута), когда выполняется следующее условие: если $\left(a_{1}, \ldots, a_{n}\right) \in A^{n}$, то $f\left(a_{1}, \ldots, a_{n}\right) \in A$.

Определение 6 (Самодвойственность). Называем функцию $f$ самодвойственной относительно перестановки $\iota$ на $A$, когда $f\left(a_{1}, \ldots, a_{n}\right)=$ $\iota f\left(\iota a_{1}, \ldots, \iota a_{n}\right)$. 
Известно, что $E_{4}-$ класс всех функций, сохраняющих $\{\mathbf{t}, \mathbf{f}\}$ Avron, 1999, Th. 3.15]. Демонстрация предполноты $\operatorname{Tr}$ в $E_{4}$ сводится к обоснованию двух утверждений: (1) $T r$ - класс всех функций, которые одновременно $\{\mathbf{t}, \mathbf{f}\}$-замкнуты и самодвойственны относительно $-x ;(2)$ класс всех функций, которые одновременно $\{\mathbf{t}, \mathbf{f}\}$-замкнуты и самодвойственны относительно $-x$, предполон в классе всех функций, сохраняющих $\{\mathbf{t}, \mathbf{f}\}$.

Введем ряд вспомогательных обозначений: $J_{\mathbf{t}}(x)=x \wedge-x ; J_{\mathbf{f}}(x)=$ $\neg J_{\mathbf{t}}(x) ; \bullet x=\neg\left(J_{\mathbf{t}}(x) \vee J_{\mathbf{f}}(x)\right)$. Указанные операции отвечают таблицам, приведенным ниже.

\begin{tabular}{|c|c|c|c|c|c|}
\hline$x$ & $J_{\mathbf{t}}(x)$ & $x$ & $J_{\mathbf{f}}(x)$ & $x$ & • $(x)$ \\
\hline $\mathbf{t}$ & $t$ & $\mathrm{t}$ & $\mathrm{f}$ & $\mathrm{t}$ & $f$ \\
\hline b & f & b & f & b & $\mathbf{t}$ \\
\hline $\mathbf{n}$ & f & $\mathbf{n}$ & f & $\mathbf{n}$ & $\mathbf{t}$ \\
\hline f & f & f & $\mathbf{t}$ & f & f \\
\hline
\end{tabular}

Теорема 1. Tr - класс всех функиий, которые одновременно $\{\mathbf{t}, \mathbf{f}\}$ замкнуты и самодвойственны относительно $-x$.

\section{Доказательство.}

Пусть $\psi\left(x_{1}, \ldots, x_{n}\right)$ - произвольная функция, сохраняющая множество $\{\mathbf{t}, \mathbf{f}\}$ и самодовойственная относительно $-x$, т. е. $\psi\left(x_{1}, \ldots, x_{n}\right)=$ $-\psi\left(-x_{1}, \ldots,-x_{n}\right)$. Пусть $\vec{a}=\left(a_{1}, \ldots, a_{n}\right), \vec{a} \in\{\mathbf{t}, \mathbf{b}, \mathbf{n}, \mathbf{f}\}^{n}$ и $\psi(\vec{a})=\mathbf{t}$. Так как $\psi\left(x_{1}, \ldots, x_{n}\right)=-\psi\left(-x_{1}, \ldots,-x_{n}\right)$, верно также, что $-\psi(-\vec{a})=\psi(-\vec{a})=$ $\mathbf{t}$ для набора $-\vec{a}=\left(-a_{1}, \ldots,-a_{n}\right)$.

Сделаем небольшое отступление, чтобы разъяснить используемую ниже нотацию. Если дана функция $\psi\left(x_{1}, \ldots, x_{n}\right)$, зависящая от $n$ переменных, и такой набор значений $\vec{a}=\left(a_{1}, \ldots, a_{n}\right)$, что $\vec{a} \in\{\mathbf{t}, \mathbf{b}, \mathbf{n}, \mathbf{f}\}^{n}$, осуществим разбиение $\left(x_{1}, \ldots, x_{n}\right)$ на четыре упорядоченных лексикографически набора в зависимости от того, какое значение $a_{i}$ соответствует переменной $x_{i}$ в наборе $\vec{a}$ для каждого из $i \in\{1, \ldots, n\}$ : если $a_{i}=\mathbf{t}$, то $x_{i}$ является компонентом $\vec{x}_{t}$; если $a_{i}=\mathbf{b}$, то $x_{i}$ является компонентом $\vec{x}_{b}$; если $a_{i}=\mathbf{n}$, то $x_{i}$ является компонентом $\vec{x}_{n}$, если $a_{i}=\mathbf{f}$, то $x_{i}$ является компонентом $\vec{x}_{f}$.

Проиллюстрируем описанное построение примером. Для этого рассмотрим функцию $\psi\left(x_{1}, x_{2}, x_{3}, x_{4}, x_{5}, x_{6}, x_{7}, x_{8}\right)$ и набор $\vec{a}=(\mathbf{t}, \mathbf{t}, \mathbf{b}, \mathbf{n}, \mathbf{b}, \mathbf{b}, \mathbf{n}, \mathbf{f})$. В этом случае имеем $\vec{x}_{t}=\left(x_{1}, x_{2}\right) ; \vec{x}_{b}=\left(x_{3}, x_{5}, x_{6}\right) ; \vec{x}_{n}=\left(x_{4}, x_{7}\right) ; \vec{x}_{f}=\left(x_{8}\right)$. При обобщенном описании будем использовать для элементов $\vec{x}_{t}, \vec{x}_{b}, \vec{x}_{n}$, $\vec{x}_{f}$ индексы вида $i_{t}, i_{b}, i_{n}, i_{f}$ соответственно. Эти индексы трактуем как переменные для исходных индексов, использованных в наборе $\left(x_{1}, \ldots, x_{n}\right)$. То есть в случае нашего примера, $x_{1_{t}}=x_{1} ; x_{2_{t}}=x_{2} ; x_{1_{b}}=x_{3} ; x_{2_{b}}=x_{5}$; $x_{3_{b}}=x_{6} ; x_{1_{n}}=x_{4} ; x_{2_{n}}=x_{7} ; x_{1_{n}}=x_{8}$. Порядок индексов (буквы под цифрами) должен быть именно таким, поскольку наборы индексов $\left(t_{1}, \ldots t_{k}\right)$, 
$\left(b_{1}, \ldots b_{k}\right),\left(n_{1}, \ldots n_{k}\right)$ и $\left(f_{1}, \ldots f_{k}\right)$ полагаются равномощными, в отличие от наборов $\left(1_{t}, \ldots k_{t}\right),\left(1_{b}, \ldots k_{b}\right),\left(1_{n}, \ldots k_{n}\right)$ и $\left(1_{f}, \ldots k_{f}\right)$. Так как наборы могут быть разной длины, в индексах для последнего элемента набора используется метапеременная $k$. То есть в случае нашего примера, $x_{k_{t}}=x_{2_{t}}$; $x_{k_{b}}=x_{3_{b}} ; x_{k_{n}}=x_{2_{n}} ; x_{f_{n}}=x_{1_{n}}$.

Так как $\left(x_{1}, \ldots, x_{n}\right)$ - список переменных, от которых зависит $\psi$, все элементы в нем попарно различны. Таким образом, всегда попарно различны и элементы $\vec{x}_{t}, \vec{x}_{b}, \vec{x}_{n}, \vec{x}_{f}$. Для каждого из этих наборов будем обозначать число его элементов как $\left|\vec{x}_{t}\right|,\left|\vec{x}_{b}\right|,\left|\vec{x}_{n}\right|,\left|\vec{x}_{f}\right|$ соответственно.

Возвращаемся к построению доказательства. Рассмотрим формулу $\Phi_{\vec{a}}^{t}\left(x_{1}, \ldots, x_{n}\right)$, которая реализует функцию $\Phi_{\vec{a}}^{t}$ :

$$
\Phi_{\vec{a}}^{t}\left(x_{1}, \ldots, x_{n}\right)=\Delta\left(\vec{x}_{t}\right) \wedge \Theta\left(\vec{x}_{f}\right) \wedge \Xi\left(\vec{x}_{b}, \vec{x}_{n}\right),
$$

Условимся считать, что обозначения $\Delta\left(\vec{x}_{t}\right)=\emptyset, \Theta\left(\vec{x}_{f}\right)=\emptyset$ и $\Xi\left(\vec{x}_{b}, \vec{x}_{n}\right)=\emptyset$ служат метаязыковыми указаниями на необходимость опустить один или более конъюнктов при построении формулы $\Phi_{\vec{a}}^{t}\left(x_{1}, \ldots, x_{n}\right)$. Например, если $\Delta\left(\vec{x}_{t}\right)=\emptyset, \Theta\left(\vec{x}_{t}\right) \neq \emptyset, \Xi\left(\vec{x}_{t}\right) \neq \emptyset$, то $\Phi_{\vec{a}}^{t}\left(x_{1}, \ldots, x_{n}\right)$ принимает вид $\Theta\left(\vec{x}_{f}\right) \wedge \Xi\left(\vec{x}_{b}, \vec{x}_{n}\right)$. Если же $\Delta\left(\vec{x}_{t}\right)=\emptyset, \Theta\left(\vec{x}_{t}\right)=\emptyset, \Xi\left(\vec{x}_{t}\right) \neq \emptyset$, то $\Phi_{\vec{a}}^{t}\left(x_{1}, \ldots, x_{n}\right)$ принимает вид $\Xi\left(\vec{x}_{b}, \vec{x}_{n}\right)$, и так далее. Обратим внимание, что каждый набор $\vec{a}$ содержит по меньшей мере одно истинностое значение, поэтому хотя бы один конъюнкт в $\Phi_{\vec{a}}^{t}\left(x_{1}, \ldots, x_{n}\right)$ всегда остается на месте. Переходим к расшифровке $\Delta\left(\vec{x}_{t}\right), \Theta\left(\vec{x}_{f}\right)$ и $\Xi\left(\vec{x}_{b}, \vec{x}_{n}\right)$.

$$
\begin{aligned}
& \Delta\left(\vec{x}_{t}\right)=\left\{\begin{array}{l}
\emptyset, \text { если }\left|\vec{x}_{t}\right|=0, \\
J_{\mathbf{t}}\left(x_{1_{t}}\right), \text { если }\left|\vec{x}_{t}\right|=1, \\
J_{\mathbf{t}}\left(x_{1_{t}} \wedge \ldots \wedge x_{k_{t}}\right), \text { если }\left|\vec{x}_{t}\right|=k_{t} \geqslant 2 .
\end{array}\right. \\
& \Theta\left(\vec{x}_{f}\right)=\left\{\begin{array}{l}
\emptyset, \text { если }\left|\vec{x}_{f}\right|=0, \\
J_{\mathbf{f}}\left(x_{1_{f}}\right), \text { если }\left|\vec{x}_{f}\right|=1, \\
J_{\mathbf{f}}\left(x_{1_{f}} \vee \ldots \vee x_{k_{t}}\right), \text { если }\left|\vec{x}_{f}\right|=k_{f} \geqslant 2 .
\end{array}\right.
\end{aligned}
$$

Функция $\Xi\left(\vec{x}_{b}, \vec{x}_{n}\right)$ описывается приведенной ниже таблицей, где $\tilde{x}$ обозначает $x \wedge \neg x$. 


\begin{tabular}{|c|c|c|}
\hline$\left|\vec{x}_{b}\right|$ & $\left|\vec{x}_{n}\right|$ & $\Xi\left(\vec{x}_{b}, \vec{x}_{n}\right)$ \\
\hline 0 & 0 & $\emptyset$ \\
1 & 0 & $\bullet\left(x_{1_{b}}\right)$ \\
$k_{b} \geqslant 2$ & 0 & $\bullet\left(\tilde{x}_{1_{b}} \wedge \ldots \wedge \tilde{x}_{k_{b}}\right)$ \\
0 & 1 & $\bullet\left(x_{1_{n}}\right)$ \\
1 & 1 & $\bullet\left(x_{1_{b}} \wedge-x_{1_{n}}\right)$ \\
$k_{b} \geqslant 2$ & 1 & $\bullet\left(\left(\tilde{x}_{1_{b}} \wedge \ldots \wedge \tilde{x}_{k_{b}}\right) \wedge-\tilde{x}_{1_{n}}\right)$ \\
0 & $k_{n} \geqslant 2$ & $\bullet\left(\tilde{x}_{1_{n}} \wedge \ldots \wedge \tilde{x}_{k_{n}}\right)$ \\
1 & $k_{n} \geqslant 2$ & $\bullet\left(\tilde{x}_{1_{b}} \wedge-\left(\tilde{x}_{1_{n}} \wedge \ldots \wedge \tilde{x}_{k_{n}}\right)\right)$ \\
$k_{b} \geqslant 2$ & $k_{n} \geqslant 2$ & $\bullet\left(\left(\tilde{x}_{1_{b}} \wedge \ldots \wedge \tilde{x}_{k_{b}}\right) \wedge-\left(\tilde{x}_{1_{n}} \wedge \ldots \wedge \tilde{x}_{k_{n}}\right)\right)$ \\
\hline
\end{tabular}

По построению, $\Phi_{\vec{a}}^{t}$ принимает значение $\mathbf{t}$ на наборах $\vec{a}$ и $-\vec{a}$, а на всех остальных наборах она принимает значение $\mathbf{f}$.

Пусть $T=\left\{\vec{a}_{1}, \ldots, \vec{a}_{k}\right\}-$ множество всех таких наборов, что $\psi\left(\overrightarrow{a_{i}}\right)=\mathbf{t}$ для всех $i \in\{1, \ldots, k\}$. Функция $\Phi_{T}^{t}=\Phi_{\vec{a}_{1}}^{t} \vee \ldots \vee \Phi_{\vec{a}_{k}}^{t}$ принимает значение $\mathbf{t}$ на каждом наборе значений из $T$ и принимает значение $\mathbf{f}$ во всех остальных случаях.

Теперь пусть $\vec{a}=\left(a_{1}, \ldots, a_{n}\right), \vec{a} \in\{\mathbf{t}, \mathbf{b}, \mathbf{n}, \mathbf{f}\}^{n}$ и $\psi(\vec{a})=\mathbf{b}$. Так как $\psi\left(x_{1}, \ldots, x_{n}\right)=-\psi\left(-x_{1}, \ldots,-x_{n}\right)$, верно также, что $\psi(-\vec{a})=\mathbf{n}$ для набора $-\vec{a}=\left(-a_{1}, \ldots,-a_{n}\right)$ (см. выше). Аналогично, если $\psi(\vec{a})=\mathbf{n}$, то $\psi(-\vec{a})=$ $\mathbf{b}$. То есть класс $B \subseteq\{\mathbf{t}, \mathbf{b}, \mathbf{n}, \mathbf{f}\}^{n}$ всех наборов, на которых $\psi$ принимает значение $\mathbf{b}$, и класс $N \subseteq\{\mathbf{t}, \mathbf{b}, \mathbf{n}, \mathbf{f}\}^{n}$ всех наборов, на которых $\psi$ принимает значение $\mathbf{n}$, изоморфны относительно $-x$.

Рассмотрим функцию, реализуемую формулой $\Phi_{\vec{a}}^{b}\left(x_{1}, \ldots, x_{n}\right)$ :

$$
\Phi_{\vec{a}}^{b}\left(x_{1}, \ldots, x_{n}\right)=\Delta\left(\vec{x}_{t}\right) \wedge \Theta\left(\vec{x}_{f}\right) \wedge \Xi\left(\vec{x}_{b}, \vec{x}_{n}\right),
$$

где $\Delta\left(\vec{x}_{t}\right)$ и $\Theta\left(\vec{x}_{f}\right)$ определяются так же, как для $\Phi_{\vec{a}}^{t}$, а функция $\Xi\left(\vec{x}_{b}, \vec{x}_{n}\right)$ описывается приведенной ниже таблицей. Обратим внимание, что $\left|\vec{x}_{b}\right| \neq 0$ или $\left|\vec{x}_{n}\right| \neq 0$, так как $\psi$ сохраняет множество $\{\mathbf{t}, \mathbf{f}\}$.

\begin{tabular}{|c|c|c|}
\hline$\left|\vec{x}_{b}\right|$ & $\left|\vec{x}_{n}\right|$ & $\Xi\left(\vec{x}_{b}, \vec{x}_{n}\right)$ \\
\hline 1 & 0 & $\tilde{x}_{1_{b}}$ \\
$k_{b} \geqslant 2$ & 0 & $\tilde{x}_{1_{b}} \wedge \ldots \wedge \tilde{x}_{k_{b}}$ \\
0 & 1 & $\tilde{x}_{1_{n}}$ \\
1 & 1 & $\tilde{x}_{1_{b}} \wedge-\tilde{x}_{1_{n}}$ \\
$k_{b} \geqslant 2$ & 1 & $\left(\tilde{x}_{1_{b}} \wedge \ldots \wedge \tilde{x}_{k_{b}}\right) \wedge-\tilde{x}_{1_{n}}$ \\
0 & $k_{n} \geqslant 2$ & $\tilde{x}_{1_{n}} \wedge \ldots \wedge \tilde{x}_{k_{n}}$ \\
1 & $k_{n} \geqslant 2$ & $\tilde{x}_{1_{b}} \wedge-\left(\tilde{x}_{1_{n}} \wedge \ldots \wedge \tilde{x}_{k_{n}}\right)$ \\
$k_{b} \geqslant 2$ & $k_{n} \geqslant 2$ & $\left(\tilde{x}_{1_{b}} \wedge \ldots \wedge \tilde{x}_{k_{b}}\right) \wedge-\left(\tilde{x}_{1_{n}} \wedge \ldots \wedge \tilde{x}_{k_{n}}\right)$ \\
\hline
\end{tabular}

По построению, $\Phi_{\vec{a}}^{b}$ принимает значение $\mathbf{b}$ на наборе $\vec{a}$ и значение $\mathbf{n}$ на наборе $-\vec{a}$, а на всех остальных наборах она принимает значение $\mathbf{f}$. 
В силу изоморфизма множеств $B$ и $N$, имеем следующее. Функция $\Phi_{B}^{b}=\Phi_{\vec{a}_{1}}^{b} \vee \ldots \vee \Phi_{\vec{a}_{k}}^{b}$ принимает значение $\mathbf{b}$ на каждом наборе значений из $B$, принимает значение $\mathbf{n}$ на каждом наборе значений из $N$ и принимает значение $\mathbf{f}$ во всех остальных случаях. Так как $\mathbf{f} \vee a=a$ для любого $a \in\{\mathbf{t}, \mathbf{b}, \mathbf{n}, \mathbf{f}\}$, получаем: $\psi=\Phi_{T}^{t} \vee \Phi_{B}^{b}$.

Таким образом, каждая функция, которая одновременно $\{\mathbf{t}, \mathbf{f}\}$ замкнута и самодвойственна относительно $-x$, содержится в $\operatorname{Tr}$. Поскольку, кроме того, каждая функция из множества $\{\wedge, \vee, \neg,-\}$ также $\{\mathbf{t}, \mathbf{f}\}$ замкнута и самодвойственна относительно $-x, \operatorname{Tr}-$ класс всех функций, которые одновременно $\{\mathbf{t}, \mathbf{f}\}$-замкнуты и самодвойственны относительно $-x$.

Теорема 2. Класс $\operatorname{Tr}$ предполон в $\mathrm{E}_{4}$.

\section{Доказательство.}

Вспомним, что $\operatorname{Tr}=[\{\wedge, \vee, \neg,-\}]$ и $\iota_{4}=[\{\wedge, \vee, \neg,-, \supset\}]$ (см. стр. 120). Кроме того, имеет место равенство $x \supset y=\neg e_{2}(x) \vee y$. Таким образом, для доказательства теоремы достаточно обосновать следующее утверждение: если $\varphi \notin \operatorname{Tr}$ и $\varphi \in L_{4}$, то $e_{2} \in[\operatorname{Tr} \cup\{\varphi\}]$.

Согласно Теореме 1, $\operatorname{Tr}$ - класс всех функций, которые одновременно $\{\mathbf{t}, \mathbf{f}\}$-замкнуты и самодвойственны относительно $-x ; E_{4}-$ класс всех функций, сохраняющих $\{\mathbf{t}, \mathbf{f}\}$.

Пусть $\varphi-$ произвольная $\{\mathbf{t}, \mathbf{f}\}$-замкнутая функция, не самодовойственная относительно $-x$, и формула $\varphi\left(x_{1}, \ldots, x_{n}\right)$ реализует функцию $\varphi$. По условию, найдется такой набор $\left(a_{1}, \ldots, a_{n}\right) \in\{\mathbf{t}, \mathbf{b}, \mathbf{n}, \mathbf{f}\}^{n}$, что $\varphi\left(a_{1}, \ldots, a_{n}\right) \neq-\varphi\left(-a_{1}, \ldots,-a_{n}\right)$. Произведем такую подстановку $\epsilon$ в $\varphi\left(x_{1}, \ldots, x_{n}\right)$, что для каждой переменной $x_{i}(1 \leqslant i \leqslant n)$ имеет место

$$
\epsilon\left(x_{i}\right)=\left\{\begin{array}{l}
\neg\left(J_{\mathbf{t}}(x) \wedge \neg J_{\mathbf{t}}(x)\right), \text { если } a_{i}=\mathbf{t} ; \\
x, \text { если } a_{i}=\mathbf{b} ; \\
-x, \text { если } a_{i}=\mathbf{n} ; \\
J_{\mathbf{t}}(x) \wedge \neg J_{\mathbf{t}}(x), \text { если } a_{i}=\mathbf{f} .
\end{array}\right.
$$

Формула $\varphi\left(\epsilon\left(x_{1}\right), \ldots, \epsilon\left(x_{n}\right)\right)$ реализует функцию $\varphi^{*}$ от одной переменной, которая $\{\mathbf{t}, \mathbf{f}\}$-замкнута и не самодовойственна относительно $-x$, так как, по построению, $\varphi^{*}(\mathbf{b}) \neq-\varphi^{*}(-\mathbf{b})$. Поскольку $-\mathbf{b}=\mathbf{n}$, также имеем $\varphi^{*}(\mathbf{b}) \neq-\varphi^{*}(\mathbf{n})$. Теперь разберем случаи.

1. $\varphi^{*}(\mathbf{b})=\mathbf{t},-\varphi^{*}(\mathbf{n}) \neq \mathbf{t}$.

$$
\text { (a) }-\varphi^{*}(\mathbf{n})=\mathbf{b}, \varphi^{*}(\mathbf{n})=\mathbf{n} ; J_{\mathbf{n}} x=\bullet \varphi^{*}(x) .
$$


(b) $-\varphi^{*}(\mathbf{n})=\mathbf{n}, \varphi^{*}(\mathbf{n})=\mathbf{b} ; J_{\mathbf{n}} x=\bullet \varphi^{*}(x)$.

(c) $-\varphi^{*}(\mathbf{n})=\mathbf{f}, \varphi^{*}(\mathbf{n})=\mathbf{f} ; J_{\mathbf{n}} x=\neg\left(\neg \bullet x \vee \varphi^{*}(x)\right)$.

2. $\varphi^{*}(\mathbf{b})=\mathbf{b},-\varphi^{*}(\mathbf{n}) \neq \mathbf{b}$.

(a) $-\varphi^{*}(\mathbf{n})=\mathbf{t}, \varphi^{*}(\mathbf{n})=\mathbf{t} ; J_{\mathbf{b}} x=\bullet \varphi^{*}(x)$.

(b) $-\varphi^{*}(\mathbf{n})=\mathbf{n}, \varphi^{*}(\mathbf{n})=\mathbf{b} ; J_{\mathbf{b}} x=\bullet\left(\varphi^{*}(x) \vee(x \vee \neg x)\right)$.

(c) $-\varphi^{*}(\mathbf{n})=\mathbf{f}, \varphi^{*}(\mathbf{n})=\mathbf{f} ; J_{\mathbf{b}} x=\bullet \varphi^{*}(x)$.

3. $\varphi^{*}(\mathbf{b})=\mathbf{n},-\varphi^{*}(\mathbf{n}) \neq \mathbf{n}$.

(a) $-\varphi^{*}(\mathbf{n})=\mathbf{t}, \varphi^{*}(\mathbf{n})=\mathbf{t} ; J_{\mathbf{b}} x=\bullet \varphi^{*}(x)$.

(b) $-\varphi^{*}(\mathbf{n})=\mathbf{b}, \varphi^{*}(\mathbf{n})=\mathbf{n} ; J_{\mathbf{b}} x=\bullet\left(\varphi^{*}(x) \wedge(x \wedge \neg x)\right)$.

(c) $-\varphi^{*}(\mathbf{n})=\mathbf{f}, \varphi^{*}(\mathbf{n})=\mathbf{f} ; J_{\mathbf{b}} x=\bullet \varphi^{*}(x)$.

4. $\varphi^{*}(\mathbf{b})=\mathbf{f},-\varphi^{*}(\mathbf{n}) \neq \mathbf{f}$.

(a) $-\varphi^{*}(\mathbf{n})=\mathbf{t}, \varphi^{*}(\mathbf{n})=\mathbf{t} ; J_{\mathbf{n}} x=\bullet x \wedge \varphi^{*}(x)$.

(b) $-\varphi^{*}(\mathbf{n})=\mathbf{b}, \varphi^{*}(\mathbf{n})=\mathbf{n} ; J_{\mathbf{n}} x=\bullet \varphi^{*}(x)$.

(c) $-\varphi^{*}(\mathbf{n})=\mathbf{n}, \varphi^{*}(\mathbf{n})=\mathbf{b} ; J_{\mathbf{n}} x=\bullet \varphi^{*}(x)$.

Нетрудно убедиться, что выполняются следующие условия: $J_{\mathbf{b}}(x) \in$ $\{\mathbf{t}, \mathbf{f}\}$ и $J_{\mathbf{b}}(x)=\mathbf{t} \Leftrightarrow x=\mathbf{b} ; J_{\mathbf{n}}(x) \in\{\mathbf{t}, \mathbf{f}\}$ и $J_{\mathbf{n}}(x)=\mathbf{t} \Leftrightarrow x=\mathbf{n}$.

Теперь покажем, что $e_{2} \in\left[\left\{\wedge, \vee, \neg,-, J_{\mathbf{b}}\right\}\right]$ и $e_{2} \in\left[\left\{\wedge, \vee, \neg,-, J_{\mathbf{n}}\right\}\right]$. Для этого достаточно следующих тождеств: $e_{2}(x)=J_{\mathbf{t}}(x) \vee J_{\mathbf{b}}(x) ; e_{2}(x)=$ $\neg\left(J_{\mathbf{n}}(x) \vee J_{\mathbf{f}}(x)\right)$. Теорема доказана.

\section{2. Надклассы $T^{\prime \prime}$, предполные в $\ell_{4}$}

В этом разделе мы демонстрируем, что между $T^{\prime \prime}$ и $\ell_{4}$ лежит ровно два замкнутых класса, причем $T^{\prime \prime}$ предполон в каждом из этих классов, и оба этих класса предполны в $E_{4}$.

Теорема 3. $T^{\prime \prime}$ - класс всех функиий, сохраняющих множества $\{\mathbf{t}, \mathbf{f}\}$, $\{\mathbf{t}, \mathbf{b}, \mathbf{f}\} u,\{\mathbf{t}, \mathbf{n}, \mathbf{f}\}$.

\section{Доказательство.}

Известно, что $[\{\neg, \wedge, \supset, \mathbf{f}\}]$ есть в точности класс всех функций, обладающих каждым из следующих свойств: $\{\mathbf{t}, \mathbf{b}, \mathbf{f}\}$-замкнутость, $\{\mathbf{t}, \mathbf{n}, \mathbf{f}\}$ замкнутость, $\{\mathbf{t}, \mathbf{f}\}$-замкнутость Arieli, Avron, 1998, Th. 14]. Поэтому достаточно доказать, что $T^{\prime \prime}=[\{\neg, \wedge, \supset, \mathbf{f}\}]$.

Поскольку операции $\wedge, \vee, \neg, e_{2}$ обладают каждым из трех указанных свойств, для завершения доказательства достаточно следующих тождеств: $\mathbf{f}=e_{2}(x) \wedge \neg e_{2}(x) ; x \supset y=\neg e_{2}(x) \vee y$. 
Как следствие, между $T^{\prime \prime}$ и $E_{4}$ лежат по меньшей мере два замкнутых класса - класс $T^{\star}$ всех функций, сохраняющих множества $\{\mathbf{t}, \mathbf{f}\}$ и $\{\mathbf{t}, \mathbf{n}, \mathbf{f}\}$, а также класс $T^{\star \star}$ всех функций, сохраняющих множества $\{\mathbf{t}, \mathbf{f}\}$ и $\{\mathbf{t}, \mathbf{b}, \mathbf{f}\}$.

Теорема 4. Класс $T^{\prime \prime}$ предполон в $T^{\star}$.

\section{Доказательство.}

Достаточно доказать следующее утверждение: если $\sigma \notin T^{\prime \prime}$ и $\sigma \in T^{\star}$, то $\left[T^{\prime \prime} \cup\{\sigma\}\right]=T^{\star}$.

Пусть $\sigma$ - функция, которая сохраняет множества $\{\mathbf{t}, \mathbf{f}\}$ и $\{\mathbf{t}, \mathbf{n}, \mathbf{f}\}$ и не сохраняет множество $\{\mathbf{t}, \mathbf{b}, \mathbf{f}\}$, и формула $\sigma\left(x_{1}, \ldots, x_{n}\right)$ реализует функцию $\sigma$. По условию, найдется такой набор $\left(a_{1}, \ldots, a_{n}\right) \in\{\mathbf{t}, \mathbf{b}, \mathbf{f}\}^{n}$, что $\sigma\left(a_{1}, \ldots, a_{n}\right)=\mathbf{n}$. Произведем такую подстановку $\epsilon$ в $\sigma\left(x_{1}, \ldots, x_{n}\right)$, что для каждой переменной $x_{i}(1 \leqslant i \leqslant n)$ имеет место

$$
\epsilon\left(x_{i}\right)=\left\{\begin{array}{l}
\neg\left(J_{\mathbf{t}}(x) \wedge \neg J_{\mathbf{t}}(x)\right), \text { если } a_{i}=\mathbf{t} ; \\
x, \text { если } a_{i}=\mathbf{b} ; \\
J_{\mathbf{t}}(x) \wedge \neg J_{\mathbf{t}}(x), \text { если } a_{i}=\mathbf{f} .
\end{array}\right.
$$

Формула $\sigma\left(\epsilon\left(x_{1}\right), \ldots, \epsilon\left(x_{n}\right)\right)$ реализует функцию $\sigma^{*}(x)$ от одной переменной, которая отвечает следующим условиям: $\sigma^{*}(x) \in\{\mathbf{t}, \mathbf{n}, \mathbf{f}\}, x=\mathbf{b} \Rightarrow$ $\sigma^{*}(x)=\mathbf{n}$.

Известно, что $T^{\prime \prime}=\left[\left\{\neg, \wedge, \vee, J_{\mathbf{t}}, J_{\mathbf{b}}, J_{\mathbf{n}}, J_{\mathbf{f}}\right\}\right]$ Karpenko, 2013, §3]. Как следствие, $J_{\mathbf{b}}(x) \wedge \sigma^{*}(x) \in\left[T^{\prime \prime} \cup\{\sigma\}\right]$.

Обозначим $J_{\mathbf{b}}(x) \wedge \sigma^{*}(x)$ как $\star x$. Имеет место следующее:

$$
\star x=\left\{\begin{array}{l}
\mathbf{n}, \text { если } x=\mathbf{b} ; \\
\mathbf{f} \text { в противном случае. }
\end{array}\right.
$$

Теперь покажем, что $T^{\star}=\left[\left\{\neg, \wedge, \supset, J_{\mathbf{t}}, J_{\mathbf{b}}, J_{\mathbf{n}}, J_{\mathbf{f}}, \star\right\}\right]$.

Пусть $\psi\left(x_{1}, \ldots, x_{n}\right)$ - произвольная функция, сохраняющая множества $\{\mathbf{t}, \mathbf{f}\}$ и $\{\mathbf{t}, \mathbf{n}, \mathbf{f}\}$. Пусть $\vec{a}=\left(a_{1}, \ldots, a_{n}\right), \vec{a} \in\{\mathbf{t}, \mathbf{b}, \mathbf{n}, \mathbf{f}\}^{n}$ и $\psi(\vec{a})=\mathbf{t}$.

Определим функцию $\Phi_{\vec{a}}^{t}$ для набора $\vec{a}$ следующим образом: $\Phi_{\vec{a}}^{t}\left(x_{1}, \ldots, x_{n}\right)=J_{i_{1}}\left(x_{1}\right) \wedge \ldots \wedge J_{i_{n}}\left(x_{n}\right)$, где $i_{j}=a_{j}$. В силу определений $\wedge, J_{\mathbf{t}}, J_{\mathbf{b}}, J_{\mathbf{n}}, J_{\mathbf{f}}$,

$$
\Phi_{\vec{a}}^{t}\left(x_{1}, \ldots, x_{n}\right)=\left\{\begin{array}{l}
\mathbf{t}, \text { если } x_{j}=a_{j} \text { для всех } j \in\{1, \ldots, n\} ; \\
\mathbf{f} \text { в противном случае. }
\end{array}\right.
$$

Пусть $T=\left\{\vec{a}_{1}, \ldots, \vec{a}_{k}\right\}-$ множество всех таких наборов, что $\psi\left(\overrightarrow{a_{i}}\right)=\mathbf{t}$ для всех $i \in\{1, \ldots, k\}$. Функция $\Phi_{T}^{t}=\Phi_{\vec{a}_{1}}^{t} \vee \ldots \vee \Phi_{\vec{a}_{k}}^{t}$ принимает значение $\mathbf{t}$ 
на каждом наборе значений из $T$ и принимает значение $\mathbf{f}$ во всех остальных случаях.

Теперь пусть $\vec{a}=\left(a_{1}, \ldots, a_{n}\right), \vec{a} \in\{\mathbf{t}, \mathbf{b}, \mathbf{n}, \mathbf{f}\}^{n}$ и $\psi(\vec{a})=\mathbf{b}$.

Определим функцию $\Phi_{\vec{a}}^{b}$ для набора $\vec{a}$ следующим образом: $\Phi_{\vec{a}}^{b}\left(x_{1}, \ldots, x_{n}\right)=\phi_{1}\left(x_{1}\right) \wedge \ldots \wedge \phi_{n}\left(x_{n}\right)$, где для каждого $i \in\{1, \ldots, n\}$

$$
\phi_{i}\left(x_{i}\right)=\left\{\begin{array}{l}
x_{i} \wedge J_{\mathbf{b}}\left(x_{i}\right), \text { если } a_{i}=\mathbf{b} ; \\
J_{a_{i}}\left(x_{i}\right), \text { если } a_{i} \in\{\mathbf{t}, \mathbf{n}, \mathbf{f}\} .
\end{array}\right.
$$

Согласно условию, $\psi$ сохраняет $\{\mathbf{t}, \mathbf{n}, \mathbf{f}\}$, поэтому значение $\mathbf{b}$ встречается в наборе $\vec{a}$ по меньшей мере один раз. Это означает, в силу определений $J$-операторов и конъюнкции, что функция $\Phi_{\vec{a}}^{b}$ принимает значение b на наборе $\vec{a}$ и принимает значение $\mathbf{f}$ на всех прочих наборах.

Пусть $B=\left\{\vec{a}_{1}, \ldots, \vec{a}_{k}\right\}-$ множество всех таких наборов, что $\psi\left(\overrightarrow{a_{i}}\right)=\mathbf{b}$ для всех $i \in\{1, \ldots, k\}$. Так как $\mathbf{f} \vee \mathbf{b}=\mathbf{b}$, функция $\Phi_{B}^{b}=\Phi_{\vec{a}_{1}}^{b} \vee \ldots \vee$ $\Phi_{\vec{a}_{k}}^{b}$ принимает значение $\mathbf{b}$ на каждом наборе значений из $B$ и принимает значение $\mathbf{f}$ в любом другом случае.

Теперь пусть $\vec{a}=\left(a_{1}, \ldots, a_{n}\right), \vec{a} \in\{\mathbf{t}, \mathbf{b}, \mathbf{n}, \mathbf{f}\}^{n}$ и $\psi(\vec{a})=\mathbf{n}$.

Определим функцию $\Phi_{\vec{a}}^{n}$ для набора $\vec{a}$ следующим образом: $\Phi_{\vec{a}}^{n}\left(x_{1}, \ldots, x_{n}\right)=\phi_{1}^{\prime}\left(x_{1}\right) \wedge \ldots \wedge \phi_{n}^{\prime}\left(x_{n}\right)$, где для каждого $i \in\{1, \ldots, n\}$

$$
\phi_{i}^{\prime}\left(x_{i}\right)=\left\{\begin{array}{l}
\star x_{i}, \text { если } a_{i}=\mathbf{b} ; \\
x_{i} \wedge J_{\mathbf{n}}\left(x_{i}\right), \text { если } a_{i}=\mathbf{n} ; \\
J_{a_{i}}\left(x_{i}\right), \text { если } a_{i} \in\{\mathbf{t}, \mathbf{f}\} .
\end{array}\right.
$$

По условию теоремы, $\psi$ сохраняет множества $\{\mathbf{t}, \mathbf{f}\}$ и $\{\mathbf{t}, \mathbf{n}, \mathbf{f}\}$, однако может и не сохранять множество $\{\mathbf{t}, \mathbf{b}, \mathbf{f}\}$. Это означает, что $\vec{a}$ содержит по меньшей мере одно вхождение $\mathbf{n}$ или $\mathbf{b}$. В силу определений $J$-операторов, конъюнкции и оператора $\star x$, функция $\Phi_{\vec{a}}^{n}$ принимает значение $\mathbf{n}$ на наборе $\vec{a}$ и принимает значение $\mathbf{f}$ на всех прочих наборах.

Пусть $N=\left\{\vec{a}_{1}, \ldots, \vec{a}_{k}\right\}$ - множество всех таких наборов, что $\psi\left(\overrightarrow{a_{i}}\right)=\mathbf{n}$ для всех $i \in\{1, \ldots, k\}$. Так как $\mathbf{f} \vee \mathbf{n}=\mathbf{n}$, функция $\Phi_{N}^{n}=\Phi_{\vec{a}_{1}}^{n} \vee \ldots \vee$ $\Phi_{\vec{a}_{k}}^{n}$ принимает значение $\mathbf{n}$ на каждом наборе значений из $N$ и принимает значение $\mathbf{f}$ в любом другом случае.

Так как $\mathbf{f} \vee a=a$ для любого $a \in\{\mathbf{t}, \mathbf{b}, \mathbf{n}, \mathbf{f}\}$, получаем $\psi=$ $\Phi_{T}^{t} \vee \Phi_{B}^{b} \vee \Phi_{N}^{n}$. Таким образом, $T^{\star} \subseteq\left[\left\{\vee, \wedge, J_{\mathbf{t}}, J_{\mathbf{b}}, J_{\mathbf{n}}, J_{\mathbf{f}}, \star\right\}\right]$. Кроме того, ясно, что $\left[\left\{\vee, \wedge, J_{\mathbf{t}}, J_{\mathbf{b}}, J_{\mathbf{n}}, J_{\mathbf{f}}, \star\right\}\right] \subseteq T^{\star}$, поскольку каждая операция из $\left\{\vee, \wedge, J_{\mathbf{t}}, J_{\mathbf{b}}, J_{\mathbf{n}}, J_{\mathbf{f}}, \star\right\}$ сохраняет множества $\{\mathbf{t}, \mathbf{f}\}$ и $\{\mathbf{t}, \mathbf{n}, \mathbf{f}\}$. 
Теорема 5. Класс $T^{\star}$ предполон в $亡_{4}$.

\section{Доказательство.}

Класс $T^{\star}$ - это класс всех функций, которые сохраняют множества $\{\mathbf{t}, \mathbf{f}\}$ и $\{\mathbf{t}, \mathbf{n}, \mathbf{f}\} . E_{4}-$ класс всех функций, которые сохраняют множество $\{\mathbf{t}, \mathbf{f}\}$. Достаточно обосновать следующее утверждение: если $\varphi \notin T^{\star}$ и $\varphi \in$ $E_{4}$, то $-x \in\left[T^{\star} \cup\{\varphi\}\right]$.

Пусть $\varphi-$ функция, которая сохраняет множество $\{\mathbf{t}, \mathbf{f}\}$, но не сохраняет множество $\{\mathbf{t}, \mathbf{n}, \mathbf{f}\}$, и формула $\varphi\left(x_{1}, \ldots, x_{n}\right)$ реализует функцию $\varphi$. По условию, найдется такой набор $\left(a_{1}, \ldots, a_{n}\right) \in\{\mathbf{t}, \mathbf{n}, \mathbf{f}\}^{n}$, что $\varphi\left(a_{1}, \ldots, a_{n}\right)=\mathbf{b}$. Произведем такую подстановку $\epsilon$ в $\varphi\left(x_{1}, \ldots, x_{n}\right)$, что для каждой переменной $x_{i}(1 \leqslant i \leqslant n)$ имеет место

$$
\epsilon\left(x_{i}\right)=\left\{\begin{array}{l}
\neg\left(J_{\mathbf{t}}(x) \wedge \neg J_{\mathbf{t}}(x)\right), \text { если } a_{i}=\mathbf{t} ; \\
x, \text { если } a_{i}=\mathbf{n} ; \\
J_{\mathbf{t}}(x) \wedge \neg J_{\mathbf{t}}(x), \text { если } a_{i}=\mathbf{f} .
\end{array}\right.
$$

Формула $\varphi\left(\epsilon\left(x_{1}\right), \ldots, \epsilon\left(x_{n}\right)\right)$ реализует функцию $\varphi^{*}(x)$ от одной переменной, которая отвечает следующим условиям: $\varphi^{*}(x) \in\{\mathbf{t}, \mathbf{b}, \mathbf{f}\}, x=\mathbf{n} \Rightarrow$ $\varphi^{*}(x)=\mathbf{b}$. Теперь для завершения доказательства достаточно следующего тождества: $-x=\left(J_{\mathbf{n}}(x) \wedge \varphi^{*}(x)\right) \vee J_{\mathbf{t}}(x) \vee \star x$.

Теорема 6. Класс $T^{\prime \prime}$ предполон в $T^{\star \star}$.

Доказательство. Аналогично Теореме 4 .

Теорема 7. Класс $T^{\star \star}$ предполон в $E_{4}$.

Доказательство. Аналогично Теореме 5 .

Теорема 8. Если $T^{\prime \prime} \subset K \subseteq E_{4} u K \notin\left\{T^{\star}, T^{\star \star}\right\}$, mo $K=E_{4}$.

\section{Доказательство.}

Пусть $T^{\prime \prime} \subset K \subseteq E_{4}$ и $K \notin\left\{T^{\star}, T^{\star \star}\right\}$. Тогда $K$ содержит (необязательно различные) функции $\sigma$ и $\varphi$, которые отвечают следующим условиям: $\sigma$ сохраняет множество $\{\mathbf{t}, \mathbf{f}\}$, но не сохраняет множество $\{\mathbf{t}, \mathbf{b}, \mathbf{f}\} ; \varphi$ сохраняет множество $\{\mathbf{t}, \mathbf{f}\}$, но не сохраняет множество $\{\mathbf{t}, \mathbf{n}, \mathbf{f}\}$.

В этом случае, как вытекает из Теоремы 4, $K$ также содержит такую функцию $\sigma^{*}$ от одной переменной, что $\sigma^{*}(x) \in\{\mathbf{t}, \mathbf{n}, \mathbf{f}\}, x=\mathbf{b} \Rightarrow \sigma^{*}(x)=\mathbf{n}$. Кроме того, как следует из Теоремы $5, K$ содержит такую функцию $\varphi^{*}$ от одной переменной, что $\varphi^{*}(x) \in\{\mathbf{t}, \mathbf{b}, \mathbf{f}\}, x=\mathbf{n} \Rightarrow \varphi^{*}(x)=\mathbf{b}$. 
В ходе доказательства Теоремы 3 мы установили, что $T^{\prime \prime}=[\{\neg, \wedge, \supset, \mathbf{f}\}]$. Из этого вытекает, что $\left[T^{\prime \prime} \cup\{-x\}\right]=E_{4}$. Таким образом, для завершения доказательства достаточно следующего тождества: $-x=J_{\mathbf{t}}(x) \vee\left(J_{\mathbf{b}}(x) \wedge\right.$ $\left.\sigma^{*}(x)\right) \vee\left(J_{\mathbf{n}}(x) \wedge \varphi^{*}(x)\right)$.

В Разделах 1 и 2 мы рассмотрели четыре замкнутых класса: $T^{\prime \prime}, T r$, $T^{\star}$ и $T^{\star \star}$. Их объединяет интересная особенность. В статье De, Omori, 2015 рассмотрены четыре функции на $\{\mathbf{t}, \mathbf{b}, \mathbf{n}, \mathbf{f}\}$, являющиеся аналогами классического отрицания (нотация изменена):

\begin{tabular}{c|c|c|c|c} 
& $\sim_{1}$ & $\sim_{2}$ & $\sim_{3}$ & $\sim_{4}$ \\
\hline $\mathbf{t}$ & $\mathbf{f}$ & $\mathbf{f}$ & $\mathbf{f}$ & $\mathbf{f}$ \\
$\mathbf{b}$ & $\mathbf{n}$ & $\mathbf{n}$ & $\mathbf{f}$ & $\mathbf{f}$ \\
$\mathbf{n}$ & $\mathbf{b}$ & $\mathbf{t}$ & $\mathbf{b}$ & $\mathbf{t}$ \\
$\mathbf{f}$ & $\mathbf{t}$ & $\mathbf{t}$ & $\mathbf{t}$ & $\mathbf{t}$
\end{tabular}

Имеют место следующие факты: (1) $T r=\left[D M 4 \cup\left\{\sim_{1}\right\}\right] ;$ (2) $T^{\star}=[D M 4 \cup$ $\left.\left\{\sim_{2}\right\}\right] ;(3) T^{\star \star}=\left[D M 4 \cup\left\{\sim_{3}\right\}\right] ;(4) T^{\prime \prime}=\left[D M 4 \cup\left\{\sim_{4}\right\}\right]$. Утверждение $(1)$ истинно в силу тождеств $\sim_{1} x=-\neg x$ и $-x=\sim_{1} \neg x$. Утверждение (2) истинно, так как $\sim_{2}$ сохраняет множества $\{\mathbf{t}, \mathbf{f}\}$ и $\{\mathbf{t}, \mathbf{n}, \mathbf{f}\}$, но не сохраняет множество $\{\mathbf{t}, \mathbf{n}, \mathbf{f}\}$, и $e_{2}(x)=\sim_{2} \sim_{2} x$, т. е. $T^{\prime \prime} \subset\left[D M 4 \cup\left\{\sim_{2}\right\}\right]$ (см. Теорему (4). Доказательство утверждения (3) аналогично таковому для утверждения (2) с очевидными модификациями, используется Теорема 6 и тот факт, что $e_{2}(x)=\sim_{3} \sim_{3} x$. Истинность утверждения (4) подтверждают тождества $\sim_{4} x=\neg e_{2}(x)$ и $e_{2}(x)=\neg \sim_{4} x$. Таким образом, все четыре рассмотренных нами замкнутых класса порождены расширением $D M 4$ посредством одного из «классических» отрицаний.

\section{3. Наибольший общий подкласс $\operatorname{Tr}$ и $T^{\prime \prime}$}

Этот раздел посвящен наибольшему общему замкнутому подклассу $\mathrm{Tr}$ и $T^{\prime \prime}$. В [Карпенко, 2015, §7] автор рассматривает выразительные возможности тетравалентной модальной логики TML. Набор базовых операций TML имеет вид $\{\wedge, \vee, \neg, \square\}$, где $\square x=J_{\mathbf{t}} x$. А.С. Карпенко демонстрирует, что класс функций, порождаемый базовыми операциями этой логики (который мы обозначим как $T M L$ ), содержится как в $T r$, так и в $T^{\prime \prime}$.

В Omori, Sano, 2014 строится паранепротиворечивая логика с базовыми операциями $\{\wedge, \vee, \neg, \circ\}$, где о $x=J_{\mathbf{t}}(x) \vee J_{\mathbf{t}}(\neg x)$. B Omori, Sano, 2015] замкнутый класс, порожденный этим набором обозначается как $\mathcal{B D}$ 。. В то же время, $\mathcal{B D}$ о есть $T M L$, так как $J_{\mathbf{t}} x=x \wedge$ о $x$. Подобно А.С. Карпенко, Омори и Сано указывают, что $\mathcal{B D} \circ \subset T^{\prime \prime}$ и $\mathcal{B D} \circ \subset \operatorname{Tr}\left(T^{\prime \prime}\right.$ и $\operatorname{Tr}$ обозначаются как $\mathcal{B D} \Delta$ и $\mathcal{B D}$ - соответственно). 
Мы демонстрируем, что $T M L$ является не просто общим подклассом $\operatorname{Tr}$ и $T^{\prime \prime}$, а наибольшим замкнутым классом, обладающим таким свойством (Теорема 9). Кроме того, мы показываем, что $T M L$ предполон в $\operatorname{Tr}$ (Теорема 10, , а также что между $T M L$ и $T^{\prime \prime}$ лежит в точности один замкнутый класс (Теоремы 12 и 13.

Перед тем как перейти к изложению основных результатов, отметим, что, в отличие от $T r, T^{\prime \prime}, T^{\star}$ и $T^{\star \star}$, класс $T M L$ не содержит классического отрицания. Однако он содержит два отрицания так наз. литеральных паралогик, т. е. логик, которые отличаются от классической только на уровне литералов - пропозициональных переменных и их итерированных отрицаний. В статье [Томова, 2018] рассматриваются четыре подобных отрицания. Интересующие нас отвечают следующим тождествам: $\neg_{1} x=\neg J_{\mathbf{t}}(x)$; $\neg_{2} x=J_{\mathbf{t}}(\neg x)$. Поскольку также имеет место $J_{\mathbf{t}}(x)=\neg_{1} x$ и $J_{\mathbf{t}}(x)=\neg_{2} \neg x$, выполняется тождество $[\{\wedge, \vee, \neg, \neg 1\}]=[\{\wedge, \vee, \neg, \neg 2\}]=T M L$.

Теорема 9. $T M L=\left[\left\{\neg, \wedge, \vee, J_{\mathbf{t}}\right\}\right]$ есть наибольший общий подкласс $\operatorname{Tr}$ $u T^{\prime \prime}$.

\section{Доказательство.}

Необходимо и достаточно обосновать следующие утверждения:

1. Если $f \in \operatorname{Tr}$ и $f \in T^{\prime \prime}$, то $f \in T M L$.

2. Если $f \in T M L$, то $f \in T r$ и $f \in T^{\prime \prime}$.

Докажем первое утверждение. Если $f \in T r$, то $f\{\mathbf{t}, \mathbf{f}\}$-замкнута и самодвойственна относительно $-x$ (Теорема 1). Если $f \in T^{\prime \prime}$, то $f$ сохраняет множества $\{\mathbf{t}, \mathbf{f}\},\{\mathbf{t}, \mathbf{b}, \mathbf{f}$,$\} и \{\mathbf{t}, \mathbf{n}, \mathbf{f}\}$ (Теорема 3). Таким образом, для доказательства достаточно обосновать, что $T M L$ есть класс всех функций, одновременно обладающих следующими свойствами: $\{\mathbf{t}, \mathbf{f}\}$-замкнутость, $\{\mathbf{t}, \mathbf{b}, \mathbf{f}$,$\} -замкнутость, \{\mathbf{t}, \mathbf{n}, \mathbf{f}\}$-замкнутость, самодовойственность относительно $-x$.

Определим вспомогательную операцию: $x \boxplus y=J_{\mathbf{t}}(((x \wedge \neg x) \wedge \bullet y) \vee(\bullet x \wedge$ $(y \wedge \neg y))) \wedge(\bullet x \wedge(y \wedge \neg y))$. Операция $\boxplus$ отвечает следующей таблице:

\begin{tabular}{c|cccc}
$\boxplus$ & $\mathbf{t}$ & $\mathbf{b}$ & $\mathbf{n}$ & $\mathbf{f}$ \\
\hline $\mathbf{t}$ & $\mathbf{f}$ & $\mathbf{f}$ & $\mathbf{f}$ & $\mathbf{f}$ \\
$\mathbf{b}$ & $\mathbf{f}$ & $\mathbf{f}$ & $\mathbf{b}$ & $\mathbf{f}$ \\
$\mathbf{n}$ & $\mathbf{f}$ & $\mathbf{n}$ & $\mathbf{f}$ & $\mathbf{f}$ \\
$\mathbf{f}$ & $\mathbf{f}$ & $\mathbf{f}$ & $\mathbf{f}$ & $\mathbf{f}$
\end{tabular}

Рассмотрим функцию $\psi$, которая одновременно $\{\mathbf{t}, \mathbf{f}\}$-замкнута, $\{\mathbf{t}, \mathbf{b}, \mathbf{f}$,$\} -замкнута, \{\mathbf{t}, \mathbf{n}, \mathbf{f}\}$-замкнута и самодовойственна относительно 
$-x$. Пусть $\psi\left(x_{1}, \ldots, x_{n}\right)$ принимает значение $\mathbf{t}$ на наборе $\vec{a}=\left(a_{1}, \ldots, a_{n}\right)$. Так как $\psi\left(x_{1}, \ldots, x_{n}\right)=-\psi\left(-x_{1}, \ldots,-x_{n}\right), \psi\left(x_{1}, \ldots, x_{n}\right)$ также принимает значение $\mathbf{t}$ на наборе $-\vec{a}=\left(-a_{1}, \ldots,-a_{n}\right)$.

Рассмотрим функцию, реализуемую формулой $\Phi_{\vec{a}}^{t}\left(x_{1}, \ldots, x_{n}\right)$ :

$$
\Phi_{\vec{a}}^{t}\left(x_{1}, \ldots, x_{n}\right)=\Delta\left(\vec{x}_{t}\right) \wedge \Theta\left(\vec{x}_{f}\right) \wedge \Xi\left(\vec{x}_{b}, \vec{x}_{n}\right),
$$

где $\Delta$ и $\Theta$ определяются так же, как в Теореме 1 , а функция $\Xi\left(\vec{x}_{b}, \vec{x}_{n}\right)$ описывается приведенной ниже таблицей.

\begin{tabular}{|c|c|c|}
\hline$\left|\vec{x}_{b}\right|$ & $\left|\vec{x}_{n}\right|$ & $\Xi\left(\vec{x}_{b}, \vec{x}_{n}\right)$ \\
\hline 0 & 0 & $\emptyset$ \\
1 & 0 & $\bullet\left(x_{1_{b}}\right)$ \\
$k_{b} \geqslant 2$ & 0 & $\bullet\left(\tilde{x}_{1_{b}} \wedge \ldots \wedge \tilde{x}_{k_{b}}\right)$ \\
0 & 1 & $\bullet\left(x_{1_{n}}\right)$ \\
1 & 1 & $\bullet\left(x_{1_{b}} \boxplus x_{1_{n}}\right)$ \\
$k_{b} \geqslant 2$ & 1 & $\bullet\left(\left(\tilde{x}_{1_{b}} \wedge \ldots \wedge \tilde{x}_{k_{b}}\right) \boxplus \tilde{x}_{1_{n}}\right)$ \\
0 & $k_{n} \geqslant 2$ & $\bullet\left(\tilde{x}_{1_{n}} \wedge \ldots \wedge \tilde{x}_{k_{n}}\right)$ \\
1 & $k_{n} \geqslant 2$ & $\bullet\left(\tilde{x}_{1_{b}} \boxplus\left(\tilde{x}_{1_{n}} \wedge \ldots \wedge \tilde{x}_{k_{n}}\right)\right)$ \\
$k_{b} \geqslant 2$ & $k_{n} \geqslant 2$ & $\bullet\left(\left(\tilde{x}_{1_{b}} \wedge \ldots \wedge \tilde{x}_{k_{b}}\right) \boxplus\left(\tilde{x}_{1_{n}} \wedge \ldots \wedge \tilde{x}_{k_{n}}\right)\right)$ \\
\hline
\end{tabular}

По построению, $\Phi_{\vec{a}}^{t}$ принимает значение $\mathbf{t}$ на наборах $\vec{a}$ и $-\vec{a}$, а на всех прочих наборах она принимает значение $\mathbf{f}$.

Теперь пусть $\psi\left(x_{1}, \ldots, x_{n}\right)$ принимает значение $\mathbf{b}$ на наборе $\vec{a}=$ $\left(a_{1}, \ldots, a_{n}\right)$. Так как $\psi\left(x_{1}, \ldots, x_{n}\right)=-\psi\left(-x_{1}, \ldots,-x_{n}\right), \psi\left(x_{1}, \ldots, x_{n}\right)$ также принимает значение $\mathbf{n}$ на наборе $-\vec{a}=\left(-a_{1}, \ldots,-a_{n}\right)$. Поскольку $\psi$ сохраняет множества $\{\mathbf{t}, \mathbf{f}\}$ и $\{\mathbf{t}, \mathbf{n}, \mathbf{f}\}, \vec{a}$ содержит по меньшей мере одно вхождение b.

Рассмотрим функцию, реализуемую формулой $\Phi_{\vec{a}}^{b}\left(x_{1}, \ldots, x_{n}\right)$ :

$$
\Phi_{\vec{a}}^{b}\left(x_{1}, \ldots, x_{n}\right)=\Delta\left(\vec{x}_{t}\right) \wedge \Theta\left(\vec{x}_{f}\right) \wedge \Xi\left(\vec{x}_{b}, \vec{x}_{n}\right),
$$

где $\Delta$ и $\Theta$ определяются так же, как в Теореме 1, а функция $\Xi\left(\vec{x}_{b}, \vec{x}_{n}\right)$ описывается приведенной ниже таблицей.

\begin{tabular}{|c|c|c|}
\hline$\left|\vec{x}_{b}\right|$ & $\left|\vec{x}_{n}\right|$ & $\Xi\left(\vec{x}_{b}, \vec{x}_{n}\right)$ \\
\hline 1 & 0 & $\tilde{x}_{1_{b}}$ \\
$k_{b} \geqslant 2$ & 0 & $\tilde{x}_{1_{b}} \wedge \ldots \wedge \tilde{x}_{k_{b}}$ \\
1 & 1 & $x_{1_{b}} \boxplus x_{1_{n}}$ \\
$k_{b} \geqslant 2$ & 1 & $\left(\tilde{x}_{1_{b}} \wedge \ldots \tilde{x}_{k_{b}}\right) \boxplus \tilde{x}_{1_{n}}$ \\
1 & $k_{n} \geqslant 2$ & $\tilde{x}_{1_{b}} \boxplus\left(\tilde{x}_{1_{n}} \wedge \ldots \wedge \tilde{x}_{k_{n}}\right)$ \\
$k_{b} \geqslant 2$ & $k_{n} \geqslant 2$ & $\left(\tilde{x}_{1_{b}} \wedge \ldots \wedge \tilde{x}_{k_{b}}\right) \boxplus\left(\tilde{x}_{1_{n}} \wedge \ldots \wedge \tilde{x}_{k_{n}}\right)$ \\
\hline
\end{tabular}


Функция $\Phi_{\vec{a}}^{b}$ принимает значение $\mathbf{b}$ на наборе $\vec{a}$ и значение $\mathbf{n}$ на наборе $-\vec{a}$, a на всех остальных наборах она принимает значение $\mathbf{f}$.

Как и в доказательстве Теоремы 1. в силу изоморфизма множества $B$ всех наборов, на которых $\psi$ принимает значение $\mathbf{b}$, и множества $N$ всех наборов, на которых $\psi$ принимает значение $\mathbf{n}$, имеем следующее. Функция $\Phi_{B}^{b}=\Phi_{\vec{a}_{1}}^{b} \vee \ldots \vee \Phi_{\vec{a}_{k}}^{b}$ принимает значение $\mathbf{b}$ на каждом наборе значений из $B$, принимает значение $\mathbf{n}$ на каждом наборе значений из $N$ и принимает значение $\mathbf{f}$ во всех остальных случаях.

Так как $\mathbf{f} \vee a=a$ для любого $a \in\{\mathbf{t}, \mathbf{b}, \mathbf{n}, \mathbf{f}\}$, получаем $\psi=\Phi_{T}^{t} \vee \Phi_{B}^{b}$. Таким образом, каждая функция, которая одновременно $\{\mathbf{t}, \mathbf{f}\}$-замкнута, $\{\mathbf{t}, \mathbf{b}, \mathbf{f}$,$\} -замкнута, \{\mathbf{t}, \mathbf{n}, \mathbf{f}\}$-замкнута и самодовойственна относительно $-x$, содержится в $T M L$.

Покольку, кроме того, каждая функция из множества $\left\{\wedge, \vee, \neg, J_{\mathbf{t}}\right\}$ также обладает всеми из перечисленных выше свойств, $T M L$ действительно есть класс всех функций, которые одновременно $\{\mathbf{t}, \mathbf{f}\}$-замкнуты, $\{\mathbf{t}, \mathbf{b}, \mathbf{f}$,$\} -замкнуты, \{\mathbf{t}, \mathbf{n}, \mathbf{f}\}$-замкнуты и самодовойственны относительно $-x$. Из этого факта тривиальным образом следует и второе утверждение: если $f \in T M L$, то $f \in \operatorname{Tr}$ и $f \in T^{\prime \prime}$.

Теорема 10. Класс TML предполон в $\mathrm{Tr}$.

\section{Доказательство.}

Вспомним, что $T M L=\left[\left\{\neg, \wedge, \vee, J_{\mathbf{t}}\right\}\right]($ стр. 129) и $\operatorname{Tr}=[\{\wedge, \vee, \neg,-\}]$ (стр. 120). Таким образом, для доказательства теоремы достаточно обосновать следующее утверждение: если $\varphi \notin T M L$ и $\varphi \in T r$, то $-x \in$ $[T M L \cup\{\varphi\}]$.

$T M L$ - класс всех функций, одновременно обладающих следующими свойствами: $\{\mathbf{t}, \mathbf{f}\}$-замкнутость, $\{\mathbf{t}, \mathbf{b}, \mathbf{f}$,$\} -замкнутость ,\{\mathbf{t}, \mathbf{n}, \mathbf{f}\}$ замкнутость, самодовойственность относительно $-x ; \operatorname{Tr}-$ класс всех функций, которые одновременно $\{\mathbf{t}, \mathbf{f}\}$-замкнуты и самодвойственны относительно $-x$. Пусть $\varphi-$ произвольная $\{\mathbf{t}, \mathbf{f}\}$-замкнутая функция, самодовойственная относительно $-x$, которая не сохраняет множество $\{\mathbf{t}, \mathbf{b}, \mathbf{f}$, или $\{\mathbf{t}, \mathbf{n}, \mathbf{f}$,$\} , и формула \varphi\left(x_{1}, \ldots, x_{n}\right)$ реализует функцию $\varphi$.

Допустим, найдется такой набор $\left(a_{1}, \ldots, a_{n}\right) \in\{\mathbf{t}, \mathbf{b}, \mathbf{f}\}^{n}$, что $\varphi\left(a_{1}, \ldots, a_{n}\right)=\mathbf{n}$. Произведем такую подстановку $\epsilon$ в $\varphi\left(x_{1}, \ldots, x_{n}\right)$, что для каждой переменной $x_{i}(1 \leqslant i \leqslant n)$ имеет место

$$
\epsilon\left(x_{i}\right)=\left\{\begin{array}{l}
\neg\left(J_{\mathbf{t}}(x) \wedge \neg J_{\mathbf{t}}(x)\right), \text { если } a_{i}=\mathbf{t} ; \\
x, \text { если } a_{i}=\mathbf{b} ; \\
J_{\mathbf{t}}(x) \wedge \neg J_{\mathbf{t}}(x), \text { если } a_{i}=\mathbf{f} .
\end{array}\right.
$$


Формула $\varphi\left(\epsilon\left(x_{1}\right), \ldots, \epsilon\left(x_{n}\right)\right)$ реализует функцию $\varphi^{*}$ от одной переменной, которая $\{\mathbf{t}, \mathbf{f}\}$-замкнута, самодовойственна относительно $-x$ и не сохраняет множество $\{\mathbf{t}, \mathbf{b}, \mathbf{f}$,$\} . То есть \varphi^{*}(\mathbf{b})=\mathbf{n}$. В то же время, в силу самодвойственности, $\varphi^{*}(\mathbf{b})=-\varphi^{*}(-\mathbf{b})=-\varphi^{*}(\mathbf{n})$. Таким образом, $\varphi^{*}(\mathbf{n})=\mathbf{b}$. Однако тогда выполняется следующее тождество: $-x=\left(\varphi^{*}(x) \wedge \neg J_{\mathbf{t}}(x) \wedge\right.$ $\left.\neg J_{\mathbf{t}}(\neg x)\right) \vee J_{\mathbf{t}}(x)$.

Теперь допустим, что найдется такой набор $\left(a_{1}, \ldots, a_{n}\right) \in\{\mathbf{t}, \mathbf{n}, \mathbf{f}\}^{n}$, для которого $\varphi\left(a_{1}, \ldots, a_{n}\right)=\mathbf{b}$. Чтобы провести построение, аналогичное только что рассмотренному случаю, достаточно очевидной модификации определения подстановки $\epsilon: \epsilon\left(x_{i}\right)=x$, если $a_{i}=\mathbf{n}$.

Итак, мы установили, что $T M L$ предполон в $T r$. Теперь установим отношения между $T M L$ и $T^{\prime \prime}$. Для этого используем понятие сохранения матрицы функиией. Подробнее о этом можно прочитать в Марченков, 2004. $\S 1.6]$.

Определение 7 (Сохранение матрицы функцией). Пусть дана матрица ${ }^{2}$ вида $\mathfrak{M}=\left(\begin{array}{ccc}a_{11} & \ldots & a_{1 p} \\ \ldots & \ldots & \ldots \\ a_{m 1} & \ldots & a_{m p}\end{array}\right)$, где $a_{k l} \in A$ для всех $k \in\{1, \ldots, m\}, l \in$ $\{1, \ldots, p\}$, и $f\left(x_{1}, \ldots, x_{n}\right)$ - функция на $A$ от $n$ переменных. Обозначим как $\mathfrak{M}_{n}$ матрицу $\left(\begin{array}{ccc}a_{1 i_{1}} & \ldots & a_{1 i_{n}} \\ \ldots & \ldots & \ldots \\ a_{m i_{1}} & \ldots & a_{m i_{n}}\end{array}\right)$, где $i_{j} \in\{1, \ldots, p\}$ для всех $j \in\{1, \ldots, n\}$.

Говорим, что функция $f\left(x_{1}, \ldots, x_{n}\right)$ сохраняет матричу $\mathfrak{M}$, если для всех $\mathfrak{M}_{n}$ имеет место $f\left(\begin{array}{ccc}a_{1 i_{1}} & \ldots & a_{1 i_{n}} \\ \ldots & \ldots & \ldots \\ a_{m i_{1}} & \ldots & a_{m i_{n}}\end{array}\right)=\left(\begin{array}{ccc}f\left(a_{1 i_{1}}\right. & \ldots & \left.a_{1 i_{n}}\right) \\ \ldots & & \\ f\left(a_{m i_{1}}\right. & \ldots & \left.a_{m i_{n}}\right)\end{array}\right) \in \mathfrak{M}$.

Теперь рассмотрим операцию ๑, которая отвечает следующей таблице:

\begin{tabular}{c|cccc}
$\odot$ & $\mathbf{t}$ & $\mathbf{b}$ & $\mathbf{n}$ & $\mathbf{f}$ \\
\hline $\mathbf{t}$ & $\mathbf{f}$ & $\mathbf{f}$ & $\mathbf{f}$ & $\mathbf{f}$ \\
$\mathbf{b}$ & $\mathbf{f}$ & $\mathbf{f}$ & $\mathbf{t}$ & $\mathbf{f}$ \\
$\mathbf{n}$ & $\mathbf{f}$ & $\mathbf{f}$ & $\mathbf{f}$ & $\mathbf{f}$ \\
$\mathbf{f}$ & $\mathbf{f}$ & $\mathbf{f}$ & $\mathbf{f}$ & $\mathbf{f}$
\end{tabular}

Эта операция не самодвойственна относительно $-x$, так как $\mathbf{b} \odot \mathbf{n}=\mathbf{t}$, однако $\mathbf{n} \odot \mathbf{b} \neq \mathbf{t}$. В то же время все операции из набора $\{\bigvee, \wedge, \neg, \odot\}$ сохраняют матрицу $\mathfrak{S}$, где

\footnotetext{
${ }^{2}$ Как это обычно делается при исследовании замкнутых классов функций, матрица используется в качестве более удобного обозначения множества упорядоченных наборов (см. Марченков, 2004, §1.6]).
} 


$$
\mathfrak{S}=\left(\begin{array}{lll}
\mathbf{b} & \mathbf{f} & \mathbf{t} \\
\mathbf{n} & \mathbf{f} & \mathbf{t}
\end{array}\right)
$$

Приведенные ниже теоремы демонстрируют, что $T M L^{\prime}=[\{\vee, \wedge, \neg$, ○ $\}]$ является единственным замкнутым классом между $T M L$ и $T^{\prime \prime}$.

Теорема 11. $T M L^{\prime}=[\{\vee, \wedge, \neg, \odot\}]-$ - ласс всех функиий, которье содержатся в $T^{\prime \prime}$ и сохраняют матрииу $\mathfrak{S}$.

Доказательство. Если $f \in T^{\prime \prime}$, то $f$ сохраняет множества $\{\mathbf{t}, \mathbf{f}\},\{\mathbf{t}, \mathbf{b}, \mathbf{f}$, и $\{\mathbf{t}, \mathbf{n}, \mathbf{f}\}$ (Теорема 3). Пусть $\psi-$ произвольная функция, не самодовойственная относительно $-x$, которая сохраняет множества $\{\mathbf{t}, \mathbf{f}\},\{\mathbf{t}, \mathbf{b}, \mathbf{f}$, и $\{\mathbf{t}, \mathbf{n}, \mathbf{f}$,$\} , а также сохраняет матрицу \mathfrak{S}$.

Пусть $\psi\left(x_{1}, \ldots, x_{n}\right)$ принимает значение $\mathbf{t}$ на наборе $\vec{a}=\left(a_{1}, \ldots, a_{n}\right)$. Поскольку $\psi$ сохраняет матрицу $\mathfrak{S}, \psi\left(x_{1}, \ldots, x_{n}\right)$ также принимает значение $\mathbf{t}$ на наборе $-\vec{a}=\left(-a_{1}, \ldots,-a_{n}\right)$.

Рассмотрим функцию, реализуемую формулой $\Phi_{\vec{a}}^{t}\left(x_{1}, \ldots, x_{n}\right)$ :

$$
\Phi_{\vec{a}}^{t}\left(x_{1}, \ldots, x_{n}\right)=\Delta\left(\vec{x}_{t}\right) \wedge \Theta\left(\vec{x}_{f}\right) \wedge \Xi\left(\vec{x}_{b}, \vec{x}_{n}\right),
$$

где $\Delta\left(\vec{x}_{t}\right)$ и $\Theta\left(\vec{x}_{f}\right)$ определяются так же, как в Теореме 1 , а $\Xi\left(\vec{x}_{b}, \vec{x}_{n}\right)$ описывается приведенной ниже таблицей.

\begin{tabular}{|c|c|c|}
\hline$\left|\vec{x}_{b}\right|$ & $\left|\vec{x}_{n}\right|$ & $\Xi\left(\vec{x}_{b}, \vec{x}_{n}\right)$ \\
\hline 0 & 0 & $\emptyset$ \\
1 & 0 & $\bullet\left(x_{1_{b}}\right)$ \\
$k_{b} \geqslant 2$ & 0 & $\bullet\left(\tilde{x}_{1_{b}} \wedge \ldots \wedge \tilde{x}_{k_{b}}\right)$ \\
0 & 1 & $x_{1_{b}} \odot x_{1_{n}}$ \\
1 & 1 & $\left(\tilde{x}_{1_{b}} \wedge \ldots \wedge \tilde{x}_{k_{b}}\right) \odot x_{1_{n}}$ \\
$k_{b} \geqslant 2$ & 1 & $\bullet\left(\tilde{x}_{1_{n}} \wedge \ldots \wedge \tilde{x}_{k_{n}}\right)$ \\
0 & $k_{n} \geqslant 2$ & $x_{1_{b}} \odot\left(\tilde{x}_{1_{n}} \wedge \ldots \wedge \tilde{x}_{k_{n}}\right)$ \\
1 & $k_{n} \geqslant 2$ & $\left.\tilde{x}_{k_{b}}\right) \odot\left(\tilde{x}_{1_{n}} \wedge \ldots \wedge \tilde{x}_{k_{n}}\right)$ \\
$k_{b} \geqslant 2$ & $k_{n} \geqslant 2$ & $\left(\tilde{x}_{1_{b}} \wedge \ldots \wedge \tilde{x}_{k}\right)$ \\
\hline
\end{tabular}

Если один из наборов $\vec{x}_{b}, \vec{x}_{n}$ является пустым, а второй непуст, то формула $\Phi_{\vec{a}}^{t}$ принимает значение $\mathbf{t}$ на наборах $\vec{a}$ и $-\vec{a}$, а на всех остальных наборах она принимает значение $\mathbf{f}$. Если же наборы $\vec{x}_{b}, \vec{x}_{n}$ одновременно пусты или одновременно непусты, то формула $\Phi_{\vec{a}}^{t}$ принимает значение $\mathbf{t}$ на одном лишь наборе $\vec{a}$ и значение $\mathbf{f}$ на всех остальных наборах.

Пусть $T=\left\{\vec{a}_{1}, \ldots, \vec{a}_{k}\right\}$ - множество всех таких наборов, что $\psi\left(\overrightarrow{a_{i}}\right)=\mathbf{t}$ для всех $i \in\{1, \ldots, k\}$. Поскольку $\psi$ сохраняет матрицу $\mathfrak{S}$, имеет место следующее: $\psi(\vec{a})=\mathbf{t} \Leftrightarrow \psi(-\vec{a})=\mathbf{t}$ для всех $\vec{a} \in\{\mathbf{t}, \mathbf{b}, \mathbf{f}\}^{n} \cup\{\mathbf{t}, \mathbf{n}, \mathbf{f}\}^{n}$. 
Функция $\Phi_{T}^{t}=\Phi_{\vec{a}_{1}}^{t} \vee \ldots \vee \Phi_{\vec{a}_{k}}^{t}$ принимает значение $\mathbf{t}$ на каждом наборе значений из $T$ и принимает значение $\mathbf{f}$ во всех остальных случаях.

Теперь пусть $\vec{a}=\left(a_{1}, \ldots, a_{n}\right), \vec{a} \in\{\mathbf{t}, \mathbf{b}, \mathbf{n}, \mathbf{f}\}^{n}$ и $\psi(\vec{a})=\mathbf{b}$. Поскольку $\psi$ сохраняет множество $\{\mathbf{t}, \mathbf{n}, \mathbf{f}\}, \vec{a}$ содержит по меньшей мере одно вхождение $\mathbf{b}$.

Определим функцию $\Phi_{\vec{a}}^{b}$ для набора $\vec{a}$ следующим образом:

$$
\Phi_{\vec{a}}^{b}\left(x_{1}, \ldots, x_{n}\right)=\Delta\left(\vec{x}_{t}\right) \wedge \Theta\left(\vec{x}_{f}\right) \wedge \Xi\left(\vec{x}_{b}, \vec{x}_{n}\right)
$$

где $\Delta\left(\vec{x}_{t}\right)$ и $\Theta\left(\vec{x}_{f}\right)$ определяются так же, как и выше, а $\Xi\left(\vec{x}_{b}, \vec{x}_{n}\right)$ описывается приведенной ниже таблицей.

\begin{tabular}{|c|c|c|}
\hline$\left|\vec{x}_{b}\right|$ & $\left|\vec{x}_{n}\right|$ & $\Xi\left(\vec{x}_{b}, \vec{x}_{n}\right)$ \\
\hline 1 & 0 & $\tilde{x}_{1_{b}}$ \\
$k_{b} \geqslant 2$ & 0 & $\tilde{x}_{1_{b}} \wedge \ldots \wedge \tilde{x}_{k_{b}}$ \\
1 & 1 & $\tilde{x}_{1_{b}} \wedge\left(x_{1_{b}} \odot x_{1_{n}}\right)$ \\
$k_{b} \geqslant 2$ & 1 & $\left(\tilde{x}_{1_{b}} \wedge \ldots \tilde{x}_{k_{b}}\right) \wedge\left(x_{1_{b}} \odot x_{1_{n}}\right)$ \\
1 & $k_{n} \geqslant 2$ & $\tilde{x}_{1_{b}} \wedge\left(x_{1_{b}} \odot x_{1_{n}} \wedge \ldots \wedge x_{1_{b}} \odot x_{k_{n}}\right)$ \\
$k_{b} \geqslant 2$ & $k_{n} \geqslant 2$ & $\left(\tilde{x}_{1_{b}} \wedge \ldots \wedge \tilde{x}_{k_{b}}\right) \wedge\left(x_{1_{b}} \odot x_{1_{n}} \wedge \ldots \wedge x_{1_{b}} \odot x_{k_{n}}\right)$ \\
\hline
\end{tabular}

Если $\left|\vec{x}_{n}\right|=0$, то формула $\Phi_{\vec{a}}^{b}$ принимает значение $\mathbf{b}$ на наборе $\vec{a}$ и значение $\mathbf{n}$ на наборе $-\vec{a}$, а на всех остальных наборах она принимает значение $\mathbf{f}$. Если $\left|\vec{x}_{n}\right| \neq 0$, то формула $\Phi_{\vec{a}}^{b}$ принимает значение $\mathbf{b}$ на одном лишь наборе $\vec{a}$ и значение $\mathbf{f}$ на всех остальных наборах.

Пусть $B=\left\{\vec{a}_{1}, \ldots, \vec{a}_{k}\right\}$ - множество всех таких наборов, что $\psi\left(\overrightarrow{a_{i}}\right)=\mathbf{b}$ для всех $i \in\{1, \ldots, k\}$, а $N=\left\{\vec{a}_{1}^{\prime}, \ldots, \vec{a}_{k}^{\prime}\right\}$ - множество всех таких наборов, что $\psi\left(\overrightarrow{a_{i}}\right)=\mathbf{n}$ для всех $i \in\{1, \ldots, k\}$. Функция $\Phi_{B}^{b}=\Phi_{\vec{a}_{1}}^{b} \vee \ldots \vee \Phi_{\vec{a}_{k}}^{b}$ принимает значение $\mathbf{b}$ на каждом наборе значений из $B$, принимает значение $\mathbf{n}$ на каждом таком наборе $\vec{a}^{\prime}$ из $N$, что $\vec{a}^{\prime} \in\{\mathbf{t}, \mathbf{n}, \mathbf{f}\}^{n}$, и принимает значение f во всех остальных случаях.

Наконец, пусть $\vec{a}=\left(a_{1}, \ldots, a_{n}\right), \vec{a} \in\{\mathbf{t}, \mathbf{b}, \mathbf{n}, \mathbf{f}\}^{n}$ и $\psi(\vec{a})=\mathbf{n}$. Поскольку $\psi$ сохраняет множество $\{\mathbf{t}, \mathbf{b}, \mathbf{f}\}^{n}, \vec{a}$ содержит по меньшей мере одно вхождение $\mathbf{n}$.

Определим функцию $\Phi_{\vec{a}}^{n}$ для набора $\vec{a}$ следующим образом:

$$
\Phi_{\vec{a}}^{n}\left(x_{1}, \ldots, x_{n}\right)=\Delta\left(\vec{x}_{t}\right) \wedge \Theta\left(\vec{x}_{f}\right) \wedge \Xi\left(\vec{x}_{b}, \vec{x}_{n}\right),
$$

где $\Delta\left(\vec{x}_{t}\right)$ и $\Theta\left(\vec{x}_{f}\right)$ определяются так же, как и выше, а $\Xi\left(\vec{x}_{b}, \vec{x}_{n}\right)$ описывается приведенной ниже таблицей. 


\begin{tabular}{|c|c|c|}
\hline$\left|\vec{x}_{b}\right|$ & $\left|\vec{x}_{n}\right|$ & $\Xi\left(\vec{x}_{b}, \vec{x}_{n}\right)$ \\
\hline 0 & 1 & $\tilde{x}_{1_{n}}$ \\
1 & 1 & $\left(x_{1_{b}} \odot x_{1_{n}}\right) \wedge \tilde{x}_{1_{n}}$ \\
$k_{b} \geqslant 2$ & 1 & $\left(\left(x_{1_{b}} \odot x_{1_{n}}\right) \wedge \ldots \wedge\left(x_{k_{b}} \odot x_{1_{n}}\right)\right) \wedge \tilde{x}_{1_{n}}$ \\
0 & $k_{n} \geqslant 2$ & $\tilde{x}_{1_{n}} \wedge \ldots \wedge \tilde{x}_{k_{n}}$ \\
1 & $k_{n} \geqslant 2$ & $\left(x_{1_{b}} \odot x_{1_{n}}\right) \wedge\left(\tilde{x}_{1_{n}} \wedge \ldots \wedge \tilde{x}_{k_{n}}\right)$ \\
$k_{b} \geqslant 2$ & $k_{n} \geqslant 2$ & $\left(\left(x_{1_{b}} \odot x_{1_{n}}\right) \wedge \ldots \wedge\left(x_{k_{b}} \odot x_{1_{n}}\right)\right) \wedge\left(\tilde{x}_{1_{n}} \wedge \ldots \wedge \tilde{x}_{k_{n}}\right)$ \\
\hline
\end{tabular}

Если $\left|\vec{x}_{b}\right|=0$, то формула $\Phi_{\vec{a}}^{n}$ принимает значение $\mathbf{n}$ на наборе $\vec{a}$ и значение $\mathbf{b}$ на наборе $-\vec{a}$, а на всех остальных наборах она принимает значение $\mathbf{f}$. Если $\left|\vec{x}_{b}\right| \neq 0$, то формула $\Phi_{\vec{a}}^{n}$ принимает значение $\mathbf{n}$ на одном лишь наборе $\vec{a}$ и значение $\mathbf{f}$ на всех остальных наборах.

Функция $\Phi_{N}^{b}=\Phi_{\vec{a}_{1}}^{n} \vee \ldots \vee \Phi_{\vec{a}_{k}}^{n}$ принимает значение $\mathbf{n}$ на каждом наборе значений из $N$, принимает значение $\mathbf{b}$ на каждом таком наборе $\vec{a}^{\prime}$ из $B$, что $\vec{a}^{\prime} \in\{\mathbf{t}, \mathbf{b}, \mathbf{f}\}^{n}$, и принимает значение $\mathbf{f}$ во всех остальных случаях.

Так как $\mathbf{f} \vee a=a$ для всех $a \in\{\mathbf{t}, \mathbf{b}, \mathbf{n}, \mathbf{f}\}$, получаем $\psi=\Phi_{T}^{b} \vee \Phi_{B}^{b} \vee \Phi_{N}^{b}$.

Теорема 12. Класс TML предполон в $T M L^{\prime}$.

Доказательство. Вспомним, что $T M L=\left[\left\{\neg, \wedge, \vee, J_{\mathbf{t}}\right\}\right]$ (стр. 129) и $T M L^{\prime}=\left[\left\{\neg, \wedge, \vee, J_{\mathbf{t}}, \odot\right\}\right]$ (стр. 134). Таким образом, для доказательства теоремы достаточно обосновать следующее утверждение: если $\varphi \notin T M L$ и $\varphi \in T M L^{\prime}$, то $\odot \in[\operatorname{Tr} \cup\{\varphi\}]$.

$T M L$ - класс всех функций, одновременно обладающих следующими свойствами: $\{\mathbf{t}, \mathbf{f}\}$-замкнутость, $\{\mathbf{t}, \mathbf{b}, \mathbf{f}$,$\} -замкнутость, \{\mathbf{t}, \mathbf{n}, \mathbf{f}\}$ замкнутость, самодовойственность относительно $-x ; T M L^{\prime}-$ класс всех функций, которые одновременно $\{\mathbf{t}, \mathbf{f}\}$-замкнуты, $\{\mathbf{t}, \mathbf{b}, \mathbf{f}$,$\} -замкнуты,$ $\{\mathbf{t}, \mathbf{n}, \mathbf{f}\}$-замкнуты, а также сохраняют матрицу $\mathfrak{S}$.

Пусть $\varphi$ - произвольная $\{\mathbf{t}, \mathbf{f}\}$-замкнутая, $\{\mathbf{t}, \mathbf{b}, \mathbf{f}$,$\} -замкнутая, \{\mathbf{t}, \mathbf{n}, \mathbf{f}\}$ замкнутая функция, не самодовойственная относительно $-x$ и сохраняющая матрицу $\mathfrak{S}$, которую реализует формула $\varphi\left(x_{1}, \ldots, x_{n}\right)$. По условию, найдется такой набор $\left(a_{1}, \ldots, a_{n}\right) \in\{\mathbf{t}, \mathbf{b}, \mathbf{n}, \mathbf{f}\}^{n}$, что $\varphi\left(a_{1}, \ldots, a_{n}\right) \neq$ $-\varphi\left(-a_{1}, \ldots,-a_{n}\right)$. Произведем такую подстановку $\epsilon$ в $\varphi\left(x_{1}, \ldots, x_{n}\right)$, что для каждой переменной $x_{i}(1 \leqslant i \leqslant n)$ имеет место

$$
\epsilon\left(x_{i}\right)=\left\{\begin{array}{l}
\neg\left(J_{\mathbf{t}}(x) \wedge \neg J_{\mathbf{t}}(x)\right), \text { если } a_{i}=\mathbf{t} ; \\
x, \text { если } a_{i}=\mathbf{b} ; \\
y, \text { если } a_{i}=\mathbf{n} ; \\
J_{\mathbf{t}}(x) \wedge \neg J_{\mathbf{t}}(x), \text { если } a_{i}=\mathbf{f} .
\end{array}\right.
$$

Формула $\varphi\left(\epsilon\left(x_{1}\right), \ldots, \epsilon\left(x_{n}\right)\right)$ реализует функцию $\varphi^{*}$ от двух переменных, которая $\{\mathbf{t}, \mathbf{f}\}$-замкнута, $\{\mathbf{t}, \mathbf{b}, \mathbf{f}$,$\} -замкнута, \{\mathbf{t}, \mathbf{n}, \mathbf{f}\}$-замкнута, не самодовойственна относительно $-x$ и сохраняет матрицу $\mathfrak{S}$. 
Обратим внимание, что $\boxplus \in T M L$ (см. стр. 130). Пусть $\varphi^{\boxplus}(x, y)=$ $\left(\varphi^{*}(x, y) \wedge \bullet(x \boxplus y)\right)$. Тогда имеет место следующее:

$$
\varphi^{\boxplus}(x, y)=\left\{\begin{array}{l}
\varphi^{*}(x, y), \text { если }(x, y) \in\{(\mathbf{b}, \mathbf{n}),(\mathbf{n}, \mathbf{b})\} ; \\
\mathbf{f} \text { в противном случае. }
\end{array}\right.
$$

Теперь разберем случаи.

1. $\varphi^{*}(\mathbf{b}, \mathbf{n})=\mathbf{t},-\varphi^{*}(\mathbf{n}, \mathbf{b}) \neq \mathbf{t}$.

(a) $-\varphi^{*}(\mathbf{n}, \mathbf{b})=\mathbf{b}, \varphi^{*}(\mathbf{n}, \mathbf{b})=\mathbf{n} ; x \odot y=J_{\mathbf{t}}\left(\varphi^{\boxplus}(x, y)\right)$.

(b) $-\varphi^{*}(\mathbf{n}, \mathbf{b})=\mathbf{n}, \varphi^{*}(\mathbf{n}, \mathbf{b})=\mathbf{b} ; x \odot y=J_{\mathbf{t}}\left(\varphi^{\boxplus}(x, y)\right)$.

(c) $-\varphi^{*}(\mathbf{n}, \mathbf{b})=\mathbf{f}, \varphi^{*}(\mathbf{n}, \mathbf{b})=\mathbf{f} ; x \odot y=J_{\mathbf{t}}\left(\varphi^{\boxplus}(x, y)\right)$.

2. $\varphi^{*}(\mathbf{b}, \mathbf{n})=\mathbf{b},-\varphi^{*}(\mathbf{n}, \mathbf{b}) \neq \mathbf{b}$.

(a) $-\varphi^{*}(\mathbf{n}, \mathbf{b})=\mathbf{t}, \varphi^{*}(\mathbf{n}, \mathbf{b})=\mathbf{t} ; x \odot y=J_{\mathbf{t}}\left(\varphi^{\boxplus}(y, x)\right)$.

(b) $-\varphi^{*}(\mathbf{n}, \mathbf{b})=\mathbf{n}, \varphi^{*}(\mathbf{n}, \mathbf{b})=\mathbf{b} ; x \odot y=\neg J_{\mathbf{f}}\left(\varphi^{\boxplus}(x, y) \wedge(x \boxplus y)\right)$.

(c) $-\varphi^{*}(\mathbf{n}, \mathbf{b})=\mathbf{f}, \varphi^{*}(\mathbf{n}, \mathbf{b})=\mathbf{f} ; x \odot y=J_{\mathbf{f}}\left(\varphi^{\boxplus}(y, x)\right)$.

3. $\varphi^{*}(\mathbf{b}, \mathbf{n})=\mathbf{n},-\varphi^{*}(\mathbf{n}, \mathbf{b}) \neq \mathbf{n}$.

(a) $-\varphi^{*}(\mathbf{n}, \mathbf{b})=\mathbf{t}, \varphi^{*}(\mathbf{n}, \mathbf{b})=\mathbf{t} ; x \odot y=J_{\mathbf{t}}\left(\varphi^{\boxplus}(y, x)\right)$.

(b) $-\varphi^{*}(\mathbf{n}, \mathbf{b})=\mathbf{b}, \varphi^{*}(\mathbf{n}, \mathbf{b})=\mathbf{n} ; x \odot y=J_{\mathbf{t}}\left(\varphi^{\boxplus}(x, y) \vee(x \boxplus y)\right)$.

(c) $-\varphi^{*}(\mathbf{n}, \mathbf{b})=\mathbf{f}, \varphi^{*}(\mathbf{n}, \mathbf{b})=\mathbf{f} ; x \odot y=J_{\mathbf{f}}\left(\varphi^{\boxplus}(y, x)\right)$.

4. $\varphi^{*}(\mathbf{b}, \mathbf{n})=\mathbf{f},-\varphi^{*}(\mathbf{n}, \mathbf{b}) \neq \mathbf{f}$.

(a) $-\varphi^{*}(\mathbf{n}, \mathbf{b})=\mathbf{t}, \varphi^{*}(\mathbf{n}, \mathbf{b})=\mathbf{t} ; x \odot y=J_{\mathbf{f}}\left(\varphi^{\boxplus}(y, x)\right)$.

(b) $-\varphi^{*}(\mathbf{n}, \mathbf{b})=\mathbf{b}, \varphi^{*}(\mathbf{n}, \mathbf{b})=\mathbf{n} ; x \odot y=J_{\mathbf{f}}\left(\varphi^{\boxplus}(y, x)\right)$.

(c) $-\varphi^{*}(\mathbf{n}, \mathbf{b})=\mathbf{n}, \varphi^{*}(\mathbf{n}, \mathbf{b})=\mathbf{b} ; x \odot y=J_{\mathbf{f}}\left(\varphi^{\boxplus}(y, x)\right)$.

Теорема доказана.

Теорема 13. Класс $T M L^{\prime}$ предполон в $T^{\prime \prime}$.

Доказателъство. Вспомним, что $T M L^{\prime}=\left[\left\{\neg, \wedge, \vee, J_{\mathbf{t}}, \odot\right\}\right]$ (стр. 134 ) и $T^{\prime \prime}=\left[\left\{\neg, \wedge, \vee, J_{\mathbf{t}}, J_{\mathbf{b}}, J_{\mathbf{n}}, J_{\mathbf{f}}\right\}\right]$ (см. Теорему 44). Кроме того, выполняются следующие тождества: $J_{\mathbf{b}}(x)=\neg\left(J_{\mathbf{t}}(x) \vee J_{\mathbf{n}}(x) \vee J_{\mathbf{f}}(x)\right) ; J_{\mathbf{n}}(x)=$ $\neg\left(J_{\mathbf{t}}(x) \vee J_{\mathbf{b}}(x) \vee J_{\mathbf{f}}(x)\right)$. Таким образом, для доказательства теоремы достаточно обосновать следующее утверждение: если $\varphi \notin T M L^{\prime}$ и $\varphi \in T^{\prime \prime}$, то $\left\{J_{\mathbf{b}}, J_{\mathbf{n}}\right\} \cap[\operatorname{Tr} \cup\{\varphi\}] \neq \emptyset$. 
Класс $T M L^{\prime}$ - это класс всех функций, которые одновременно $\{\mathbf{t}, \mathbf{f}\}$ замкнуты, $\{\mathbf{t}, \mathbf{b}, \mathbf{f}$,$\} -замкнуты, \{\mathbf{t}, \mathbf{n}, \mathbf{f}\}$-замкнуты, а также сохраняют матрицу $\mathfrak{S} ; T^{\prime \prime}$ есть класс всех функций, которые одновременно $\{\mathbf{t}, \mathbf{f}\}$ замкнуты, $\{\mathbf{t}, \mathbf{b}, \mathbf{f}$,$\} -замкнуты и \{\mathbf{t}, \mathbf{n}, \mathbf{f}\}$-замкнуты.

Пусть $\varphi-$ произвольная $\{\mathbf{t}, \mathbf{f}\}$-замкнутая, $\{\mathbf{t}, \mathbf{b}, \mathbf{f}$,$\} -замкнутая и$ $\{\mathbf{t}, \mathbf{n}, \mathbf{f}\}$-замкнутая функция, не сохраняющая матрицу $\mathfrak{S}$, которую реализует формула $\varphi\left(x_{1}, \ldots, x_{n}\right)$. По условию, найдутся такие наборы $\left(a_{11}, \ldots, a_{1 n}\right)$ и $\left(a_{21}, \ldots, a_{2 n}\right)$, что $\varphi\left(\begin{array}{lll}a_{11} & \ldots & a_{1 n} \\ a_{21} & \ldots & a_{2 n}\end{array}\right) \notin\left(\begin{array}{lll}\mathbf{b} & \mathbf{f} & \mathbf{t} \\ \mathbf{n} & \mathbf{f} & \mathbf{t}\end{array}\right)$, однако $\left(\begin{array}{c}a_{1 i} \\ a_{2 i}\end{array}\right) \in\left(\begin{array}{lll}\mathbf{b} & \mathbf{f} & \mathbf{t} \\ \mathbf{n} & \mathbf{f} & \mathbf{t}\end{array}\right)$ для всех $i \in\{1, \ldots, n\}$.

Произведем такую подстановку $\epsilon$ в $\varphi\left(x_{1}, \ldots, x_{n}\right)$, что для каждой переменной $x_{i}(1 \leqslant i \leqslant n)$ имеет место

$$
\epsilon\left(x_{i}\right)=\left\{\begin{array}{l}
\neg\left(J_{\mathbf{t}}(x) \wedge \neg J_{\mathbf{t}}(x)\right), \text { если } a_{1 i}=a_{2 i}=\mathbf{t} ; \\
x, \text { если } a_{1 i}=\mathbf{b}, a_{2 i}=\mathbf{n} ; \\
J_{\mathbf{t}}(x) \wedge \neg J_{\mathbf{t}}(x), \text { если } a_{1 i}=a_{2 i}=\mathbf{f} .
\end{array}\right.
$$

Формула $\varphi\left(\epsilon\left(x_{1}\right), \ldots, \epsilon\left(x_{n}\right)\right)$ реализует функцию $\varphi^{*}$ от одной переменной, которая $\{\mathbf{t}, \mathbf{f}\}$-замкнута, $\{\mathbf{t}, \mathbf{b}, \mathbf{f}$,$\} -замкнута, \{\mathbf{t}, \mathbf{n}, \mathbf{f}\}$-замкнута и не сохраняет матрицу $\mathfrak{S}$. Поскольку $\varphi^{*}\{\mathbf{t}, \mathbf{b}, \mathbf{f}$,$\} -замкнута и \{\mathbf{t}, \mathbf{n}, \mathbf{f}\}$-замкнута, $\varphi^{*}(\mathbf{b}) \in\{\mathbf{t}, \mathbf{b}, \mathbf{f}\}$ и $\varphi^{*}(\mathbf{n}) \in\{\mathbf{t}, \mathbf{n}, \mathbf{f}\}$.

Теперь разберем случаи.

1. $\varphi^{*}(\mathbf{b})=\mathbf{t} ; \varphi^{*}(\mathbf{n}) \neq \mathbf{t}$;

(a) $\varphi^{*}(\mathbf{b})=\mathbf{t} ; \varphi^{*}(\mathbf{n})=\mathbf{n} ; J_{\mathbf{n}}(x)=\neg\left(J_{\mathbf{t}}\left(\varphi^{*}(x)\right) \vee J_{\mathbf{t}}(x) \vee J_{\mathbf{f}}(x)\right)$.

(b) $\varphi^{*}(\mathbf{b})=\mathbf{t} ; \varphi^{*}(\mathbf{n})=\mathbf{f} ; J_{\mathbf{n}}(x)=\neg\left(\varphi^{*}(x) \vee J_{\mathbf{t}}(x) \vee J_{\mathbf{f}}(x)\right)$.

2. $\varphi^{*}(\mathbf{b})=\mathbf{b} ; \varphi^{*}(\mathbf{n}) \neq \mathbf{n}$;

(a) $\varphi^{*}(\mathbf{b})=\mathbf{b} ; \varphi^{*}(\mathbf{n})=\mathbf{t} ; J_{\mathbf{b}}(x)=\neg\left(J_{\mathbf{t}}\left(\varphi^{*}(x)\right) \vee J_{\mathbf{t}}(x) \vee J_{\mathbf{f}}(x)\right)$.

(b) $\varphi^{*}(\mathbf{b})=\mathbf{b} ; \varphi^{*}(\mathbf{n})=\mathbf{f} ; J_{\mathbf{b}}(x)=\neg\left(J_{\mathbf{f}}\left(\varphi^{*}(x)\right) \vee J_{\mathbf{t}}(x) \vee J_{\mathbf{f}}(x)\right)$.

3. $\varphi^{*}(\mathbf{b})=\mathbf{f} ; \varphi^{*}(\mathbf{n}) \neq \mathbf{f}$;

(a) $\varphi^{*}(\mathbf{b})=\mathbf{f} ; \varphi^{*}(\mathbf{n})=\mathbf{n} ; J_{\mathbf{n}}(x)=\neg\left(J_{\mathbf{f}}\left(\varphi^{*}(x)\right) \vee J_{\mathbf{t}}(x) \vee J_{\mathbf{f}}(x)\right)$.

(b) $\varphi^{*}(\mathbf{b})=\mathbf{f} ; \varphi^{*}(\mathbf{n})=\mathbf{t} ; J_{\mathbf{b}}(x)=\neg\left(\varphi^{*}(x) \vee J_{\mathbf{t}}(x) \vee J_{\mathbf{f}}(x)\right)$.

Теорема доказана.

Поскольку в доказательстве Теоремы 13 не используется тот факт, что $\{\odot\} \in T M L$, имеем также следующее. 
Следствие 1. Если $[T M L \cup\{f\}] \subseteq T^{\prime \prime}$ и $[T M L \cup\{f\}] \nsubseteq \subseteq M L^{\prime}$, то $[T M L \cup$ $\{f\}]=T^{\prime \prime}$.

Таким образом, $T M L^{\prime}$ - единственный класс, являющийся собственным надклассом $T M L$ и собственным подклассом $T^{\prime \prime}$.

Теорема 14. Если $T M L \subset K \subseteq E_{4} u K \notin\left\{T^{\star}, T^{\star \star}, T r\right\}$, mo $K=E_{4}$.

\section{Доказательство.}

Пусть $\varphi_{1}-$ произвольная функция, которая $\{\mathbf{t}, \mathbf{f}\}$-замкнута и не $\{\mathbf{t}, \mathbf{n}, \mathbf{f}$,$\} -замкнута, т. е. \varphi_{1} \notin T^{\star} ; \varphi_{2}-$ произвольная функция, которая $\{\mathbf{t}, \mathbf{f}\}$-замкнута и не $\{\mathbf{t}, \mathbf{b}, \mathbf{f}$,$\} -замкнута, т. е. \varphi_{2} \notin T^{\star \star} ; \varphi_{3}$ - произвольная функция, которая $\{\mathbf{t}, \mathbf{f}\}$-замкнута и не самодвойственна относительно $-x$, т. е. $\varphi_{3} \notin T r$.

Существует такой набор $\left(a_{1}, \ldots, a_{n}\right) \in\{\mathbf{t}, \mathbf{n}, \mathbf{f}\}^{n}$, что $\varphi_{1}\left(a_{1}, \ldots, a_{n}\right)=\mathbf{b}$. Произведем такую подстановку $\epsilon$ в $\varphi\left(x_{1}, \ldots, x_{n}\right)$, что для каждой переменной $x_{i}(1 \leqslant i \leqslant n)$ имеет место

$$
\epsilon\left(x_{i}\right)=\left\{\begin{array}{l}
\neg\left(J_{\mathbf{t}}(x) \wedge \neg J_{\mathbf{t}}(x)\right), \text { если } a_{i}=\mathbf{t} ; \\
x, \text { если } a_{i}=\mathbf{n} ; \\
J_{\mathbf{t}}(x) \wedge \neg J_{\mathbf{t}}(x), \text { если } a_{i}=\mathbf{f} .
\end{array}\right.
$$

Формула $\varphi_{1}\left(\epsilon\left(x_{1}\right), \ldots, \epsilon\left(x_{n}\right)\right)$ реализует функцию $\varphi_{1}^{*}$ от одной переменной, которая $\{\mathbf{t}, \mathbf{f}\}$-замкнута и не $\{\mathbf{t}, \mathbf{n}, \mathbf{f}\}$-замкнута. Поскольку $\varphi_{1}^{*}\{\mathbf{t}, \mathbf{f}\}$ замкнута, $\varphi_{1}^{*}(\mathbf{t}) \in\{\mathbf{t}, \mathbf{f}\}, \varphi_{1}^{*}(\mathbf{f}) \in\{\mathbf{t}, \mathbf{f}\}$. Поскольку $\varphi_{1}^{*}$ в то же время не сохраняет множество $\{\mathbf{t}, \mathbf{n}, \mathbf{f}\}, \varphi_{1}^{*}(\mathbf{n})=\mathbf{b}$.

Допустим, что $\varphi_{1}^{*}$ самодвойственна относительно $-x$. Тогда $\varphi_{1}^{*}(\mathbf{b})=\mathbf{n}$. В этом случае имеет место следующее тождество: $-x=\varphi_{1}^{*}(x) \vee J_{\mathbf{t}}(x) \wedge$ $\neg J_{\mathbf{f}}(x)$. Вспомним, что $\operatorname{Tr}=[\{\wedge, \vee, \neg,-\}]$ (стр. 120). Это означает, что $\operatorname{Tr} \subseteq\left[T M L \cup\left\{\varphi_{1}\right\}\right]$. В силу Теоремы 2, $\left[T M L \cup\left\{\varphi_{1}, \varphi_{3}\right\}\right]=E_{4}$.

Теперь допустим, что $\varphi_{1}^{*}$ не самодвойственна относительно $-x$. Тогда $\varphi_{1}^{*}(\mathbf{b}) \in\{\mathbf{t}, \mathbf{f}\}$. В этом случае имеет место равенство $J_{\mathbf{n}}(x)=\bullet \varphi_{1}^{*}(x)$. Вспомним, что $T^{\prime \prime}=\left[\left\{\neg, \wedge, \vee, J_{\mathbf{t}}, J_{\mathbf{b}}, J_{\mathbf{n}}, J_{\mathbf{f}}\right\}\right]$ (см. Теорему 4) и $J_{\mathbf{t}} \in T M L$ (стр. $129 p$. Кроме того, $J_{\mathbf{f}}=J_{\mathbf{t}}(\neg x)$ и $J_{\mathbf{b}}(x)=\neg\left(J_{\mathbf{t}}(x) \vee J_{\mathbf{n}}(x) \vee J_{\mathbf{f}}(x)\right)$. Таким

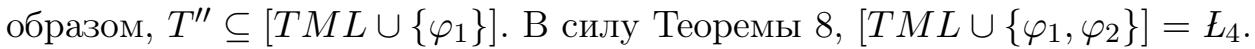

Эта теорема показывает, что $T M L$ включается в ровно три предполных класса $E_{4}-T^{\star}, T^{\star \star}$ и $T r$.

\section{Заключение}

Мы рассмотрели отношения между несколькими замкнутыми классами функций четырехзначной логики. Приведенная ниже таблица содержит сжатое резюме полученных результатов. 


\begin{tabular}{c|ccccccc} 
Свойства & $E_{4}$ & $T^{\star}$ & $T^{\star \star}$ & $T r$ & $T^{\prime \prime}$ & $T M L^{\prime}$ & $T M L$ \\
\hline$\{\mathbf{t}, \mathbf{f}\}$-замкнутость & + & + & + & + & + & + & + \\
$\{\mathbf{t}, \mathbf{n}, \mathbf{f}\}$-замкнутость & - & + & - & - & + & + & + \\
$\{\mathbf{t}, \mathbf{b}, \mathbf{f}\}$-замкнутость & - & - & + & - & + & + & + \\
Самодвойственность & - & - & - & + & - & - & + \\
Сохранение матрицы $\mathfrak{S}$ & - & - & - & - & - & + & + \\
Предполнота в $亡_{4}$ & - & + & + & + & - & - & - \\
Предполнота в $T^{\star}$ & - & - & - & - & + & - & - \\
Предполнота в $T^{\star \star}$ & - & - & - & - & + & - & - \\
Предполнота в $T r$ & - & - & - & - & - & - & + \\
Предполнота в $T^{\prime \prime}$ & - & - & - & - & - & + & - \\
Предполнота в $T M L^{\prime}$ & - & - & - & - & - & - & +
\end{tabular}

Эти результаты демонстрируют, что между $T M L$ и $E_{4}$ лежит в точности пять замкнутых классов, которые можно упорядочить по отношению вложимости, как это отражено на рис. 1 .

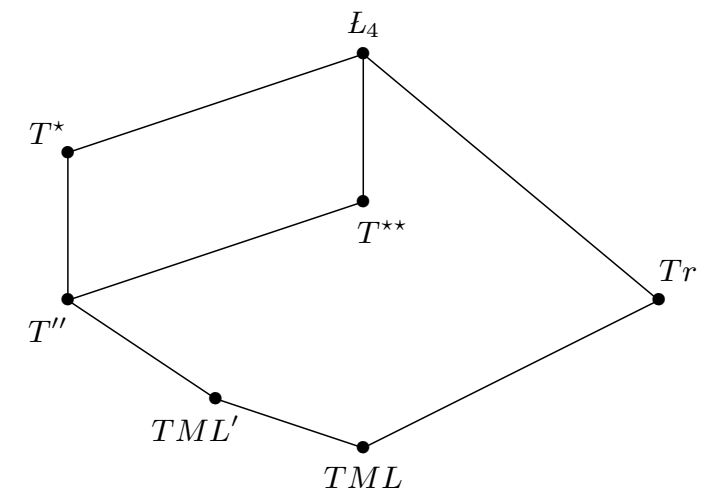

Рис. 1

Данный результат дает нам простой алгоритм, который позволяет установить выразительные возможности языка любой четырехзначной логики, если она является языковым расширением TML, и все ее операции $\{\mathbf{t}, \mathbf{f}\}$-замкнуты. Добавим к системе операций $\left\{\neg, \wedge, \vee, J_{\mathbf{t}}\right\}$ произвольную $\{\mathbf{t}, \mathbf{f}\}$-замкнутую функцию $g$, определенную на $\{\mathbf{t}, \mathbf{b}, \mathbf{n}, \mathbf{f}\}$. Тогда $\left[\left\{\neg, \wedge, \vee, J_{\mathbf{t}}, g\right\}\right] \in\left\{T M L, T M L^{\prime}, T r, T^{\prime \prime}, T^{\star}, T^{\star \star}, \iota_{4}\right\}$. Чтобы установить, с каким именно из перечисленных семи классов совпадает $\left[\left\{\neg, \wedge, \vee, J_{\mathbf{t}}, g\right\}\right]$, достаточно проверить $g$ на обладание следующими свойствами: $\{\mathbf{t}, \mathbf{f}\}$ замкнутость, $\{\mathbf{t}, \mathbf{n}, \mathbf{f}\}$-замкнутость, $\{\mathbf{t}, \mathbf{b}, \mathbf{f}\}$-замкнутость, самодвойственность относительно $-x$, сохранение матрицы $\mathfrak{S}$. 


\section{Литература}

Карпенко, 2015 - Карпенко А.C. Решетки четырехзначных модальных логик // Логические исследования. 2015. Т. 21. № 1. С. 122-137.

Марченков, 2004 - Марченков $C$. C. Функциональные системы с операцией суперпозиции. М.: ФИЗМАТЛИТ, 2004. 104 с.

Томова, 2018 - Томова Н.Е. О четырехзначных паранормальных логиках // Логические исследования. 2018. Т. 24. № 2. С. 137-143.

Avron, 1999 - Avron A. On the expressive power of three-valued and four-valued languages // Journal of Logic and Computation. 1999. Vol. 9. No. 6. P. 977-994.

Arieli, Avron, 1998 - Arieli O., Avron A. The value of the four values // Artificial Intelligence. 1998. Vol. 102. No. 1. P. 97-141.

Arieli, Avron, 2017 - Arieli O., Avron A. Four-valued paradefinite logics // Studia Logica. 2017. Vol. 105. No. 6. P. 1087-1122.

Belnap, 1977 - Belnap N. A useful four-valued logic // Modern Uses of MultipleValued Logic / Ed. by J.M. Dunn, G. Epstein. D. Reidel Publishing Co., 1977. P. 8-37.

De, Omori, 2015 - De M., Omori H. Classical Negation and Expansions of BelnapDunn Logic // Studia Logica. 2015. Vol. 103. No. 5. P. 825-851.

Karpenko, 2013 - Karpenko A.S. Von Wright's truth-logic and around // Logical Investigations. 2013. Vol. 19. P. 39-50.

Karpenko, 2017 - Karpenko A.S. Four-valued logics BD and DM4: Expansions // Bulletin of the Section of Logic. 2017. Vol. 46. No. 1-2. P. 33-45.

Omori, Sano, 2014 - Omori H., Sano K. da Costa meets Belnap and Nelson // Recent Trends in Philosophical Logic / Ed. by R. Ciuni, H. Wansing, C. Willkommen. Springer, 2014. P. 145-166.

Omori, Sano, 2015 - Omori H., Sano K. Generalizing functional completeness in Belnap-Dunn logic // Studia Logica. 2015. Vol. 103. No. 5. P. 883-917.

Omori, Wansing, 2017 - Omori H., Wansing H. 40 years of FDE: an introductory overview // Studia Logica. 2017. Vol. 105. No. 6. P. 1021-1049.

Petrukhin, Shangin, 2019 - Petrukhin Ya.I., Shangin V.O. Correspondence analysis and automated proof-searching for first degree entailment // European Journal of Mathematics. 2019. P. 1-44.

Shramko et al., 2017 - Shramko Y., Zaitsev D., Belikov A. First-Degree Entailment and its Relatives // Studia Logica. 2017). Vol. 105. P. 1291-1317.

Shramko et al., 2019 - Shramko Y., Zaitsev D., Belikov A. The FMLA-FMLA Axiomatizations of the Exactly True and Non-falsity Logics and Some of Their Cousins // Journal of Philosophical Logic. 2019. Vol. 48. P. 787-808. 
LEONID YU. DEVYATKIN

\title{
On the expressive power of certain expansions of Belnap's four-valued logic
}

\author{
Leonid Yu. Devyatkin \\ Institute of Philosophy, Russian Academy of Sciences, \\ 12/1 Goncharnaya Str., Moscow, 109240, Russian Federation. \\ E-mail: deviatkin@iph.ras.ru
}

\begin{abstract}
The paper is devoted to closed classes of functions of four-valued logic. We present the following results:

(1) The basic operations (i.e. the algebraic functions used to interpret the corresponding connectives) of the logic obtained by expanding the four-valued De Morgan algebra by the conflation operator generate the closed class of all functions that simultaneously preserve the classical truth values and are self-dual with respect to conflation. This class is precomplete in the class of all functions that preserve the classical truth values.
\end{abstract}

(2) There are exactly two closed classes between the closed class generated by the basic operations of von Wright's truth logic and the class of all functions that preserve the classical truth values. Each of them is a class of all functions that simultaneously preserve the classical truth values and one of the three-element supersets of the set of the classical truth values.

(3) The basic operations of TML, the tetravalent modal logic obtained by expanding the four-valued De Morgan algebra by the necessity operator, generate the closed class of all functions that simultaneously preserve the classical truth values, are self-dual with respect to conflation, and also preserve both three-element supersets of the set of the classical truth values. We show that this class is precomplete in the class of all functions that simultaneously preserve the classical truth values and are self-dual with respect to conflation. In addition, we demonstrate that between this class and the closed class generated by the operations of von Wright's truth logic, there is exactly one closed class.

By virtue of those results, we obtain a seven-element lattice consisting of all possible fourvalued expansions of TML that preserve classical truth values.

Keywords: De Morgan algebra, Belnap's four-valued logic, von Wright's truth logic, truth logic Tr, tetravalent modal logic, closed classes of functions

For citation: Devyatkin L.Yu. "O vyrazitel'nykh vozmozhnostyakh otdel'nykh rasshirenii chetyrekhznachnoi logiki Belnapa" [On the expressive power of certain expansions of Belnap's four-valued logic], Logicheskie Issledovaniya / Logical Investigations, 2020, Vol. 26, No. 2, pp. 116-143. DOI: 10.21146/2074-1472-2020-26-2-116-143 (In Russian) 


\section{References}

Карпенко, 2015 - Karpenko, A.S. "Reshetki chetyrekhznachnykh modal'nykh logik" [Lattices of four-valued modal logics], Logical Investigations, 2015, Vol. 21, No. 1, pp. $122-137$.

Марченков, 2004 - Marchenkov, S.S. Funktsional'nye sistemy s operatsiei superpozitsii [Functional systems with superposition operation], M., 2004. 104 p.

Томова, 2018 - Tomova, N.E. "O chetyrekhznachnykh paranormal'nykh logikakh" [On four-valued paranormal logics], Logical Investigations, 2018, Vol. 24, No. 2, pp. 137-143.

Avron, 1999 - Avron, A. "On the expressive power of three-valued and four-valued languages", Journal of Logic and Computation, 1999, Vol. 9, No. 6, pp. 977-994.

Arieli, Avron, 1998 - Arieli, O., Avron, A. "The value of the four values", Artificial Intelligence, 1998, Vol. 102, No. 1, pp. 97-141.

Arieli, Avron, 2017 - Arieli, O., Avron, A. "Four-valued paradefinite logics", Studia Logica, 2017, Vol. 105, No. 6, pp. 1087-1122.

Belnap, 1977 - Belnap, N. "A useful four-valued logic", Modern Uses of MultipleValued Logic, ed. by J.M. Dunn, G. Epstein. D. Reidel Publishing Co., 1977, pp. $8-37$.

De, Omori, 2015 - De, M., Omori, H., "Classical Negation and Expansions of BelnapDunn Logic", Studia Logica, 2015, Vol. 103, No. 5, pp. 825-851.

Karpenko, 2013 - Karpenko, A.S., "Von Wright's truth-logic and around", Logical Investigations, 2013, Vol. 19, pp. 39-50.

Karpenko, 2017 - Karpenko, A.S., "Four-valued logics BD and DM4: Expansions", Bulletin of the Section of Logic, 2017, Vol. 46, No. 1-2, pp. 33-45.

Omori, Sano, 2014 - Omori, H., Sano, K. "da Costa meets Belnap and Nelson", Recent Trends in Philosophical Logic, ed. by R. Ciuni, H. Wansing, C. Willkommen. Springer, 2014. pp. 145-166.

Omori, Sano, 2015 - Omori, H., Sano, K. "Generalizing functional completeness in Belnap-Dunn logic", Studia Logica, 2015, Vol. 103, No. 5, pp. 883-917.

Omori, Wansing, 2017 - Omori, H., Wansing, H. "40 years of FDE: an introductory overview", Studia Logica, 2017, Vol. 105, No. 6, pp. 1021-1049.

Petrukhin, Shangin, 2019 - Petrukhin, Ya.I., Shangin, V.O. "Correspondence analysis and automated proof-searching for first degree entailment", European Journal of Mathematics, 2019, pp. 1-44.

Shramko et al., 2017 - Shramko, Y., Zaitsev, D., Belikov, A. "First-Degree Entailment and its Relatives", Studia Logica, 2017, Vol. 105, pp. 1291-1317.

Shramko et al., 2019 - Shramko, Y., Zaitsev, D., Belikov, A. "The FMLA-FMLA Axiomatizations of the Exactly True and Non-falsity Logics and Some of Their Cousins", Journal of Philosophical Logic, 2019, Vol. 48, pp. 787-808. 